\title{
La composition des communautés de religieuses au Saguenay (1882-1947)
}

\section{Gérard Bouchard, Raymond Roy et Pierre Jacques}

Volume 55, 1988

Cent cinquante ans d'histoire religieuse au Saguenay-Lac-Saint-Jean (1838-1988)

URI : https://id.erudit.org/iderudit/1006949ar

DOI : https://doi.org/10.7202/1006949ar

\section{Aller au sommaire du numéro}

\section{Éditeur(s)}

Les Éditions Historia Ecclesiæ Catholicæ Canadensis Inc.

ISSN

0318-6172 (imprimé)

1927-7067 (numérique)

Découvrir la revue

Citer cet article

Bouchard, G., Roy, R. \& Jacques, P. (1988). La composition des communautés de religieuses au Saguenay (1882-1947). Sessions d'étude - Société canadienne d'histoire de l'Église catholique, 55, 87-117. https://doi.org/10.7202/1006949ar
Résumé de l'article

À partir dun répertoire biographique et à l'aide du fichier-réseau de la population du Saguenay, les auteurs étudient la composition des communautés de religieuses de cette région entre 1882 et 1947. Le texte présenté ici porte plus particulièrement sur l'évolution numérique des religieuses, leur provenance géographique à l'intérieur du Saguenay et leur origine socio-professionnelle.

Parmi les principaux résultats, on relève que :

1. Les religieuses représentent $72 \%$ de l'ensemble des gens d'Église. Elles sont, selon les sous-périodes, de quatre à six fois plus nombreuses que les prêtres. Leur recrutement commence à décroître dès la décennie 1932-1941.

2. Sur le plan spatial, toutes les parties de la région contribuent au recrutement. Il ne paraît pas exister, sous ce rapport, de stratifications locales dans l'intensité de la croyance et de la pratique. Certes, les aires de recrutement diffèrent selon les communautés; mais cette différenciation peut s'expliquer par leur emplacement et le rayonnement géographique lié à leurs fonctions.

3. De même, le recrutement ne s'alimente pas également - toutes proportions gardées - à toutes les catégories socio-professionnelles. Dans l'ensemble, les travailleurs non manuels sont légèrement sur-représentés, pendant que les ouvriers non spécialisés sont sous-représentés. Mais l'analyse par communauté démontre que ce phénomène est dans le prolongement de la différenciation spatiale.

En conclusion, à la lumière des données rapportées, on ne peut pas affirmer que les communautés pratiquaient délibérément un recrutement social sélectif.
Tous droits réservés @ Les Éditions Historia Ecclesiæ Catholicæ Canadensis Inc., 1988
Ce document est protégé par la loi sur le droit d'auteur. L’utilisation des services d'Érudit (y compris la reproduction) est assujettie à sa politique d'utilisation que vous pouvez consulter en ligne.

https://apropos.erudit.org/fr/usagers/politique-dutilisation/ 


\title{
La composition des communautés de religieuses au Saguenay (1882-1947)
}

\author{
Gérard BOUCHARD \\ Centre interuniversitaire de recherches sur les populations (SOREP) \\ Université du Québec à Chicoutimi
}

Raymond RoY

Centre interuniversitaire de recherches sur les populations (SOREP)

Université du Québec à Chicoutimi

Pierre JACQUES

Département des sciences humaines

Université du Québec à Chicoutimi

\section{Résumé}

À partir d' un répertoire biographique et à l'aide du fichier-réseau de la population du Saguenay, les auteurs étudient la composition des communautés de religieuses de cette région entre 1882 et 1947 . Le texte présenté ici porte plus particulièrement sur l'évolution numérique des religieuses, leur provenance géographique à l'intérieur du Saguenay et leur origine socio-professionnelle.

Parmi les principaux résultats, on relève que:

a) Les religieuses représentent $72 \%$ de l'ensemble des gens d'Église. Elles sont, selon les sous-périodes, de quatre à six fois plus nombreuses que les prêtres. Leur recrutement commence à décroître dès la décennie 1932-1941.

b) Sur le plan spatial, toutes les parties de la région contribuent au recrutement. Il ne paraît pas exister, sous ce rapport, de stratifications locales dans l'intensité de la croyance et de la pratique. Certes, les aires de recrutement diffèrent selon les communautés; mais cette différenciation peut s'expliquer par leur emplacement et le rayonnement géographique lié à leurs fonctions. 
c) De même, le recrutement ne s'alimente pas également - toutes proportions gardées - à toutes les catégories socio-professionnelles. Dans l' ensemble, les travailleurs non manuels sont légèrement sur-représentés, pendant que les ouvriers non spécialisés sont sous-représentés. Mais l'analyse par communauté démontre que ce phénomène est dans le prolongement de la différenciation spatiale.

En conclusion, à la lumière des données rapportées, on ne peut pas

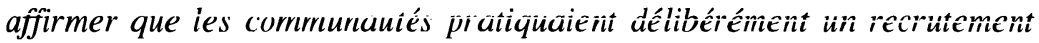
social sélectif.

\section{Introduction}

Ce texte* soumet des résultats préliminaires sur le recrutement des religieuses du Saguenay. Plus précisément, sont prises en cible les 1118 jeunes filles nées dans cette région et entrées en religion - au Saguenay ou à l'extérieur - entre 1882 et 1947 (découpage imposé par la source utilisée). L'enquête aborde les trois aspects suivants: a) l'évolution numérique des religieuses, b) leur provenance géographique, c) leur origine socio-professionnelle. Accessoirement, des données sur les clercs et sur les frères seront également présentées, mais à titre comparatif seulement. Les résultats obtenus, particulièrement en rapport avec l'évolution quantitative du recrutement, fournissent une sorte d'indicateur des mutations affectant l'Église saguenayenne au $\mathrm{XX}^{\mathrm{e}}$ siècle. Ils font voir aussi la diversité, sinon les clivages, qui existaient au sein des élites religieuses. Par ailleurs, il est utile de rappeler que la littérature sur les communautés religieuses féminines au Québec s'est enrichie sensiblement au cours des quinze dernières années. Toutefois, même si certains travaux pionniers adoptent une perspective très large (B. DENAULT, B. LÉVESQUE, 1975; M. DUMONT-JOHNSON, 1978; M. JEAN, 1977; D. BÉLANGER, L. ROZON, 1982; M. DANYLEWYCZ, 1987; D. JUNEAU, N. LAURIN, 1986), les véritables efforts de synthèse sont sans doute prématurés et il semble y avoir encore place pour des aperçus plus circonstanciés, à microéchelle, où la réalité est serrée de plus près.

Les 1118 personnes étudiées se répartissent entre plusieurs communautés religieuses. Cinq d'entre elles, regroupant près de $70 \%$ des sujets,

* La présente recherche a pu être réalisée grâce à l'assistance financière du Conseil de recherches en sciences humaines (Ottawa), du Fonds FCAR (Québec) et de l'Université du Québec à Chicoutimi. Les auteurs tiennent à remercier Régis Thibeault pour sa contribution à la compilation des données et Michel Guérin qui a confectionné les cartes. Le texte a bénéficié des commentaires formulés par Danielle Gauvreau, Guy Laperrière et Marc Saint-Hilaire, ainsi que des échanges intervenus lors d'une présentation au congrès annuel de la Société canadienne d'histoire de l'Église catholique (Chicoutimi, septembre 1988). 
ont fait en outre l'objet d'analyses plus détaillées. Nous les présentons brièvement, dans l'ordre chronologique de leur implantation au Saguenay:

- Religieuses du Bon Pasteur de Québec (Servantes du Coeur Immaculé de Marie). Fondée en 1850 à Québec, cette communauté s'implante en 1864 à Chicoutimi à la demande du curé - et futur évêque - Dominique Racine. Pendant plusieurs décennies, le couvent qu'elle fonde est la seule institution d'enseignement privé pour filles au Saguenay. Il se double d'une école normale en 1907 et dispense le cours classique à partir de 1938. Jusqu'en 1932, les activités de cette communauté se concentrent dans le Haut-Saguenay.

- Religieuses ursulines. Elles fondent en 1882, à partir de leur couvent de Québec, un monastère à Roberval (Lac-Saint-Jean) où elles se vouent à l'enseignement des jeunes filles. Leur institution comprend un pensionnat et une école ménagère agricole. Elles n'ont pas essaimé hors de Roberval pendant la période étudiée.

- Augustines hospitalières (Chanoinesses régulières hospitalières de la Miséricorde de Jésus de l'Ordre de Saint-Augustin). Arrivées à Chicoutimi en 1884, elles prennent charge de l'Hôpital de la Marine, devenu plus tard l'Hôtel-Dieu Saint-Vallier. Elles s'occupent aussi des pauvres et, jusqu'en 1926, ont charge d'un orphelinat pour filles. En 1918, elles fondent à Roberval une autre maison dont le recrutement est demeuré relativement marginal. Ces derniers effectifs ne seront pas comptés dans nos aperçus détaillés par communauté.

- Congrégation des sœurs du Bon Conseil de Chicoutimi. Fondée à Chicoutimi en 1894 sur l'initiative de Mgr M.-T. Labrecque, cette congrégation, d'abord affectée au service du clergé, s'occupe de l'enseignement primaire pour filles et garçons à partir de 1905 à l'échelle du diocèse. On la retrouve donc au Saguenay, dans Charlevoix et sur la Côte Nord, dans près d'une quarantaine de paroisses.

- Antoniennes de Marie (originellement: sœurs de Saint-Antoinede-Padoue). Cette communauté est fondée à Chicoutimi en 1904 par l'abbé Elzéar Delamarre, avec l'assistance de l'abbé Eugène Lapointe, pour remplir des fonctions auxiliaires (entretien ménager, etc.) auprès des prêtres du Séminaire et des presbytères — dans ce dernier cas à l'échelle de la région. On lui doit aussi une école pour garçons (École apostolique) destinée à promouvoir le recrutement sacerdotal. Elle essaime, elle aussi, hors du Saguenay 1 .

1 Trois communautés anciennement implantées n'ont pas été retenues pour les analyses détaillées à cause de leur faible recrutement dans ia région: les petites franciscaines de Marie (de Baie Saint-Paul, $\mathbf{N}=57$ ), les sœurs de Notre-Dame-du-Saint-Rosaire (de Rimouski, $\mathbf{N}=23)$. et les sœurs servantes du Saint-Sacrement, $(\mathbf{N}=17)$. Ces communautés ont été implantées au Saguenay à partir, respectivement, de 1928, 1894 et 1903. 
Les données de l'enquête proviennent de deux sources principales. C'est d'abord un répertoire constitué par Mgr F.-X.-E. FRENETTE (1945, 1947) et contenant le signalement de tous les clercs, religieux et religieuses originaires du diocèse de Chicoutimi, ainsi que des clercs y ayant travaillé. Le document donne a) les dates de naissance, d'entrée en religion ou d'ordination et, éventuellement, de décès, b) le nom de la communauté, quand il y a lieu, c) l'identité et, souvent, la profession des parents. Ces données, qui ont fait l'objet de diverses vérifications critiques ${ }^{2}$, ont été informatisées et intégrées (par jumelage) au fichier de population de SOREP (ou fichier BALSAC). Ce fichier constitue la deuxième source utilisée. À l'aide du système de gestion de base de données INGRID, les dossiers des religieuses ont été jumelés à leur fiche de famille, ce qui a donné accès, notamment, aux itinéraires résidentiel et socio-professionnel de leurs parents ${ }^{3}$.

Un mot enfin sur la terminologie utilisée. Suivant l'exemple de B. DENAULT et B. LÉVESQUE (1975, p. 23-24), nous emploierons le concept de communauté au sens générique, y incluant les ordres, les congrégations, les suciétés et les états religieux ${ }^{4}$. D'une manière analogue, M.-A. LESSARD et J.-P. MONTMINY (1967) ont utilisé le concept d'institut. Par ailleurs, il est utile de rappeler la distinction entre clerc et religieux. En réalité, ces deux notions se recouvrent partiellement, comme le montre la figure 1. Les religieuses, quant à elles, appartiennent à des communautés et, tout comme les frères, elles ne sont - ni clercs ni prêtres. Cependant, certains clercs sont religieux et ils relèvent d'abord de leur communauté, alors que les autres, dits séculiers, relèvent directement de l'évêque. Nous nous référerons donc ici aux prêtres (séculiers), aux religieux (prêtres ou non) et aux religieuses.

2 Notamment à l'aide d'un deuxième répertoire (A. SIMARD, 1969) contenant la liste des prêtres séculiers ayant œuvré dans le diocèse jusqu'en 1968.

3 Le fichier de population BALSAC contient les 815000 actes de l'état civil du Saguenay entre 1842 et 1986 . Il permet l'accès aux 125000 familles reconstituées, à leur généalogie et à divers fichiers dits sectoriels analogues à celui des clercs et religieux. Sur le contenu, la structure et le fonctionnement du fichier-réseau BALSAC, voir G. BOUCHARD, R. ROY, B. CASGRAIN (1985, 1986), de même que les Documents I-C-42, I-C-53 et I-C-76 de SOREP.

4 Voir les définitions canoniques résumées dans J. HAMELIN et N. GAGNON (1984, p. 144-145). 
Nomenclature des qens d'Église (a)

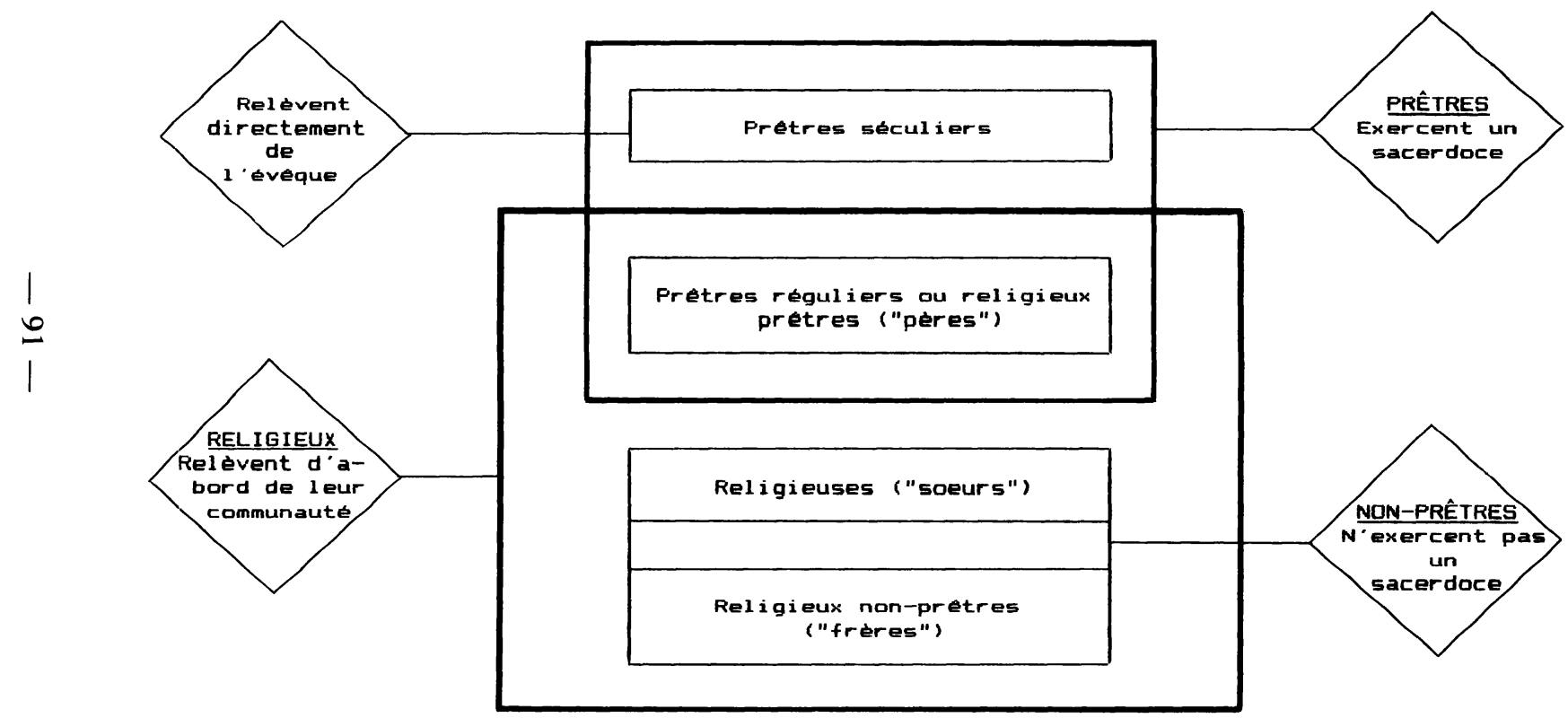

(a) Schéma réalisé à partir des définitions présentées dans A. BRIDE (1937, 1949, 1967). SOFEP 


\section{II \\ Évolution numérique des religieuses}

L'étude de l'évolution numérique des religieuses fournit d'abord un aperçu de leur poids démographique dans la société saguenayenne. Elle permet aussi d'effectuer une mesure diachronique — au moins approximative - de la foi des fidèles, dans la mesure où les vocations religieuses et sacerdotales expriment la dévotion ou la ferveur des individus et des familles. Enfin, elle fait ressortir la part représentée par les religieuses dans I'ensembie des gens d'Égiise. Cetie évoluiiun à pü êtîe quiânitifíiéc de trois façons. Une première mesure représente, pour diverses sous-périodes et pour chaque catégorie de gens d'Église, les effectifs absolus et relatifs (tableau 1). On voit que les religieuses sont de loin la catégorie la plus nombreuse. Sur toute la période, elles représentent $72 \%$ de l'ensemble des effectifs et, dans chaque sous-période, elles sont de quatre à six fois plus nombreuses que les prêtres. Leur part décroît légèrement en fin de période, surtout au profit des frères.

En deuxième lieu, la mesure du prélèvement ecclésiastique dans la population confirme que le recrutement des religieuses est en perte de vitesse en fin de période (1942-1947) (tableau 2) ${ }^{5}$. Mais c'est le cas également chez les prêtres et religieux, ce qui est confirmé par une troisième mesure, nette cette fois, du prélèvement (tableau 3). Le nombre des vocations est ici rapporté à un dénominateur beaucoup plus précis, soit l'ensemble des jeunes gens qui ont contracté un premier mariage durant chaque sous-période. Nous considérons que ce chiffre est voisin de la sous-population des «mariables» et qu'il permet donc une mesure très approchée du recrutement réel net, c'est-à-dire la proportion des jeunes gens en âge de se marier qui optaient pour la vie sacerdotale ou religieuse. On sait du reste (tableau 4) que l'âge au mariage chez les hommes et chez les femmes coïncidait avec l'âge à l'ordination ou à l'entrée en religion. Les jeunes conjoints et les candidat(e)s aux professions religieuses appartiennent donc à la même sous-population. L'indice confirme que, pour les hommes comme pour les femmes, le recrutement (c'est-à-dire les ordinations et les entrées en religion) décline après la décennie 1922-1931 et chute radicalement entre 1942 et 1947 . Pour cette dernière sous-période, on soupçonne l'influence de la Seconde Guerre mondiale, du moins en ce qui concerne le recrutement masculin. Mais on ne sait pas expliquer à ce stade-ci le ralentissement du côté féminin - nettement plus accentué que chez les hommes, rappelons-le. Se peut-il que l'effervescence économique engendrée par la guerre ait ouvert aux jeunes femmes des débouchés

5 La période 1882-1911 correspond aux fondations et implantations; ceci gonfle un peu artificiellement les chiffres du recrutement et donne l'illusion d'une chute en 1912-1921. 
Tableau 1

Prêtres, religieux et religieuses du Saguenay: évolution numérique, 1882-1947 (a)

\begin{tabular}{|c|c|c|c|c|c|c|c|c|c|c|c|c|}
\hline \multirow{2}{*}{ Catégories } & \multicolumn{10}{|c|}{ Sous-périodes } & \multicolumn{2}{|c|}{$\frac{\text { Total }}{1882-1947}$} \\
\hline & N.a. & $\%$ & N.a. & $\%$ & N.a. & $\%$ & N.a. & $\%$ & N.a. & $\%$ & N.a. & $\%$ \\
\hline Prêtres & 49 & 18,8 & 25 & 12,2 & 55 & 14,0 & 55 & 11,4 & 27 & 12,9 & 211 & 13,6 \\
\hline Religieux (pères) & 1 & 0,4 & 6 & 2,9 & 12 & 3,1 & 35 & 7,2 & 15 & 7,1 & 69 & 4,5 \\
\hline Religieuses & 199 & 76,2 & 157 & 76,6 & 298 & 76,0 & 337 & 69,6 & 127 & 60,5 & 1118 & 72,0 \\
\hline Total & 261 & 100 & 205 & 100 & 392 & 100 & 484 & 100 & 210 & 100 & 1552 & 100 \\
\hline
\end{tabular}
(a) Nés dans la région. Entrés en religion ou ordonnés entre 1882 et 1947.
(b) Sources: Mgr F.-X.-E. FRENETTE (1945, 1947).
(c) À cause des faibles effectifs, il a fallu regrouper les décennies 1882-1891, 1892-1901, 1902-1911. (SOREP) 
professionnels inexistants jusque-là? Par ailleurs, il est utile de rappeler que la décennie charnière 1932-1941 se trouve au cœur d'une période d'urbanisation très rapide au Saguenay (M. GUÉRIN, G. BOUCHARD, 1988), ce qui doit être mis en parallèle avec la chute du recrutement aussi bien masculin que féminin.

Tableau 2

Mesure brute du prélèvement sacerdotal et religieux Nombre de sujets par rapport à la population totale (a)

\begin{tabular}{|l|c|c|c|}
\hline \multirow{2}{*}{ Sous-périodes } & \multirow{2}{*}{$\begin{array}{c}\text { Population annuelle } \\
\text { moyenne de la région } \\
\text { durant chaque sous - } \\
\text { période } \\
\text { (b) }\end{array}$} & \multicolumn{2}{|c|}{ Prélèvement brut (0/00) } \\
\cline { 3 - 4 } & 38032 & Religieuses & $\begin{array}{c}\text { Prêtres et } \\
\text { religieux }\end{array}$ \\
\hline $1882-1911$ & 62115 & 5,2 & 1.6 \\
$1912-1921$ & 89547 & 2,5 & 0,8 \\
$1922-1931$ & 124582 & 3,3 & 1,1 \\
$1932-1941$ & 102329 & 2,7 & 1,2 \\
$1942-1947$ & & 1,2 & 0,8 \\
\hline
\end{tabular}

Notes: (a) Seules les personnes natives du Saguenay sont considérées. L'âge d'entrée en religion ou d'ordination a guidé le classement en souspériodes.

(b) Sources: Recensements canadiens.

Pour le reste, il se confirme que le recrutement féminin l'emporte largement sur celui des hommes (toutes catégories masculines regroupées) pour chaque sous-période, selon un rapport du simple au double en moyenne. D'un côté comme de l'autre, l'indice culmine en 1922-31, à $6,6 \%$ et $2,6 \%$ respectivement. Enfin, il est peu probable que cet écart en faveur des filles ait affecté le marché matrimonial saguenayen, les différences relevées impliquant de trop faibles effectifs ${ }^{6}$.

Il est par ailleurs indispensable de mettre les données saguenayennes en perspective à l'aide de données comparatives. Cependant, faute de

6 Du reste, la répartition par sexe des classes d'âge 20-24 et 25-30 entre 1881 et 1941 montre un équilibre à peu près constant (C. POUYEZ et alii, 1983, p. 325-326). 


\section{Tableau 3}

Mesure nette du prélèvement sacerdotal et religieux Nombre de vocations par rapport à la population des conjoints mariés au Saguenay entre 1882 et 1947 (a)

\begin{tabular}{|c|c|c|c|c|c|c|c|c|c|}
\hline \multirow[t]{2}{*}{ Sous-périodes } & \multicolumn{3}{|c|}{$\begin{array}{c}\text { (1) } \\
\text { Nombre de mariés }\end{array}$} & \multicolumn{3}{|c|}{$\begin{array}{c}(2) \\
\text { Vocations sacerdotales et } \\
\text { religieuses (c) }\end{array}$} & \multicolumn{3}{|c|}{$\begin{array}{c}\text { Prélèvement net } \\
\% \\
(2) /(1)\end{array}$} \\
\hline & Hommes & Femmes & Ensemble & Hommes (b) & Femmes & Ensemble & Hommes & Femmes & Ensemble \\
\hline $1882-1891$ & 991 & 1195 & 2186 & 13 & 31 & 44 & 1,3 & 2,6 & 2,0 \\
\hline $1892-1901$ & 1635 & 1850 & 3485 & 24 & 69 & 93 & 1,5 & 3,7 & 2,7 \\
\hline $1902-1911$ & 2292 & 2697 & 4989 & 25 & 99 & 124 & 1,1 & 3,7 & 2,5 \\
\hline $1912-1921$ & 3089 & 3718 & 6807 & 48 & 157 & 205 & 1,6 & 4,2 & 3,0 \\
\hline $1922-1931$ & 3644 & 4528 & 8172 & 94 & 298 & 392 & 2,6 & 6,6 & 4,8 \\
\hline $1932-1941$ & 6097 & 7114 & 13211 & 147 & 337 & 484 & 2,4 & 4,7 & 3,7 \\
\hline $1942-1947$ & 5735 & 7011 & 12746 & 83 & 127 & 210 & 1,5 & 1,8 & 1,7 \\
\hline $1882-1947$ & 23483 & 28113 & 51596 & 434 & 1118 & 1552 & 1,9 & 4,0 & 3,0 \\
\hline
\end{tabular}

Notes: (a) Seuls ont été pris en compte les prêtres, religieux et religieuses natifs de la région. Ils ont été classés en sous-périodes correspondant à leur âge d'entrée en religion ou à leur ordination. Parmi la population des mariés, les premières unions seulement ont été retenues.

(b) Ont été regroupés dans cette catégorie les prêtres, les pères et les frères.

(c) Dans chaque décennie le nombre de vocations est celui des ordinations et des entrées en religion durant cette période. (SOREP) 
Tableau 4

Âge des hommes et des femmes au moment de l'ordination, de l'entrée en religion (a) ou du mariage (b)

\begin{tabular}{|c|c|c|c|c|c|c|c|c|}
\hline \multirow{3}{*}{ Sous-périodes } & \multirow{2}{*}{\multicolumn{2}{|c|}{$\begin{array}{c}\begin{array}{c}\text { Âge à l'ordination } \\
\text { ou à l'entrée } \\
\text { en religion }\end{array} \\
\text { Prêtres, religieux }\end{array}$}} & \multirow{2}{*}{\multicolumn{2}{|c|}{$\begin{array}{c}\begin{array}{c}\text { Âge à } \\
\text { l'entrée en } \\
\text { religion }\end{array} \\
\text { Religieuses }\end{array}$}} & \multicolumn{4}{|c|}{ Âge au mariage } \\
\hline & & & & & \multicolumn{2}{|c|}{ Conjoints } & \multicolumn{2}{|c|}{ Conjointes } \\
\hline & N.a & Âge & N.a & $\hat{\text { Âge }}$ & N.a & Âge & N.a & $\overline{\text { Âge }}$ \\
\hline $1882-1891$ & 13 & 25,8 & 31 & 22,6 & 991 & 24,4 & 1195 & 21,4 \\
\hline $1892-1901$ & 24 & 24,6 & 69 & 23,1 & 1635 & 24,5 & 1850 & 21,4 \\
\hline $1912-1921$ & 48 & 23,3 & 157 & 21,0 & 3089 & 24,4 & 3718 & 20,8 \\
\hline $1922-1931$ & 94 & 23,5 & 298 & 20,6 & 3644 & 24,7 & 4528 & 21,0 \\
\hline $1932-1941$ & 147 & 22,7 & 337 & 20,9 & 6097 & 25,9 & 7114 & 22,5 \\
\hline $1942-1947$ & 83 & 22,2 & 127 & 20,6 & 5735 & 25,5 & 7011 & 22,4 \\
\hline $1882-1947$ & 434 & 23,2 & 1118 & 21,1 & 23483 & 25,1 & 28113 & 21,8 \\
\hline
\end{tabular}

Notes: (a) L'âge d'entrée en religion est sujet à caution puisqu'il est défini différemment selon les catégories et selon les communautés religieuses. Aussi, idéalement, il aurait fallu considérer l'âge à l'entrée au séminaire plutôt qu'à l'ordination.

(b) Seules les premières unions célébrées au cours de la période et concernant des natifs du Saguenay ont été retenues. (SOREP) 
relevés suffisants directement comparables, l'enquête ne peut malheureusement aller très loin dans cette direction. À l'aide des données produites par B. DENAULT et B. LÉVESQUE (1975, tableau 2), il est possible de confronter des taux bruts (nombre de religieuses en exercice rapporté à la population totale), mais pour deux années seulement ${ }^{7}$. Ainsi, en 1951 et 1961, ces taux bruts étaient respectivement de 7,50/00 et 6,70/00 pour la province de Québec, alors qu'ils s'établissaient à 6,20/00 et 5,50/00 pour le Saguenay. D'après l'enquête de M.-A. LESSARD et J.-P. MONTMINY (1967, tableau 13), les taux canadiens ${ }^{8}$ pour les années 1950 et 1960 seraient de 9,730/00 et 8,71 0/00. Dans les deux cas, les taux saguenayens semblent donc inférieurs; mais il faudrait aller plus loin et tenir compte de la structure de la population. Par exemple, on peut penser que les villes de Québec et Montréal, à cause du poids institutionnel associé à leur statut de métropoles, regroupaient des fonctions et des effectifs religieux proportionnellement plus nombreux que ce n'était le cas dans une région peu urbanisée comme le Saguenay. Il est donc possible que les écarts observés ne reflètent pas de véritables différences dans la croyance et dans la pratique.

À ce stade-ci, les enquêtes publiées ne permettent pas de poursuivre la comparaison sur ce plan, soit parce qu'il n'est pas possible d'isoler les religieuses des ecclésiastiques (ex.: L. PELLETIER, 1986), soit parce que les taux sont calculés d'une façon que nous ne pouvons pas reproduire avec les données saguenayennes (ex.: M. DANYLEWYCZ, 1987, p. 17).

\section{III \\ Les origines géographiques}

L'étude des lieux de provenance des religieuses à l'intérieur du Saguenay vise à mettre au jour des modalités du recrutement qui seraient associées au rayonnement géographique et, peut-être, aux fonctions spécifiques remplies par chaque communauté. Elle veut aussi faire ressortir d'éventuels clivages ou différenciations spatiales qu'on pourrait assimiler à des stratifications dans la pratique et la ferveur religieuse.

Deux notes de méthode d'abord. Pour faciliter la représentation graphique et pour éviter de traiter avec de trop petits nombres, les données ont été cartographiées à l'échelle non pas de la paroisse mais de ce que nous appelons le regroupement municipal. Il s'agit d'une unité spatiale comprenant soit une municipalité urbaine, soit quelques municipalités rurales limitrophes apparentées sur la base de la taille et des activités

7 Pour la période 1891-1941, le nombre de religieuses en exercice n'est pas connu au Saguenay.

${ }^{8}$ Les auteurs ont utilisé comme dénominateur le nombre de catholiques au Canada. 
économiques (carte 1)9. Par ailleurs, un indice comparatif a été mis au point afin de déterminer si le nombre de religieuses provenant d'un regroupement était supérieur, égal ou inférieur à la valeur attendue, compte tenu de ses effectifs démographiques. Cet indice est un rapport ayant a) comme numérateur: la proportion de l'ensemble des religieuses fournie par un regroupement, b) comme dénominateur: la proportion de la population régionale résidant dans ce regroupement. La valeur dite normale est donc égale à l'unité.

À la question d'une possible différenciation spatiale résuiltant de clivages dans l'intensité de la pratique et de la ferveur, les résultats obtenus permettent de répondre sans hésitation par la négative (carte 2). À l'exception de celles qui ont été ouvertes trop récemment au peuplement (et laissées en blanc sur la carte), toutes les parties de la région contribuent au recrutement. Quant aux inégalités observées, elles peuvent être imputées à des facteurs conjoncturels ou autres. Les zones de peuplement isolé et relativement pauvres (ex.: Saint-Honoré/Falardeau) fournissent peu, à moins qu'elles ne soient très vieilles, comme c'est le cas dans le BasSaguenay rive nord et le Bas-Saguenay rive sud. Par contre, les zones de grosses paroisses rurales prospères et bien organisées comme Métabetchouan, Normandin et Saint-Félicien, sont très prodigues. Enfin, les villes ne donnent ni plus, ni moins que la valeur attendue.

Ces remarques valent pour l'ensemble des communautés. Prises séparément, celles-ci accusent toutefois des différences importantes (tableau 5, cartes 3 à 7). Ainsi, les religieuses du Bon-Pasteur de même que les augustines hospitalières ont un recrutement beaucoup plus urbain que les autres communautés (voir l'indice comparatif pour Chicoutimi, Jonquière, La Baie). Aussi, les ursulines se recrutent surtout au sud du Lac-Saint-Jean (carte 5), pendant que le Bon-Pasteur privilégie le Haut-Saguenay. Mais nous ne possédons pas d'indice permettant d'imputer ces stratifications spatiales à des politiques préférentielles de la part des communautés. Jusqu'à plus ample informé, on peut tenir que leur implantation géographique particulière rend compte des clivages observés. Le cas du BonConseil est ici exemplaire (carte 7): c'est en même temps la communauté qui a le plus essaimé géographiquement et celle dont l'aire de recrutement était la plus dispersée.

À une échelle plus fine, on relève encore certains accidents sur lesquels il nous faudra enquêter: ainsi le fort recrutement des antoniennes à Saint-Cœur-de-Marie, celui des hospitalières dans le Bas-Saguenay rive nord, du Bon-Conseil à Normandin, ou des ursulines à Métabetchouan. Au-delà du facteur géographique évoqué plus haut, on soupçonne ici des

9 Voir à ce sujet le Document II-C-107 de SOREP. 


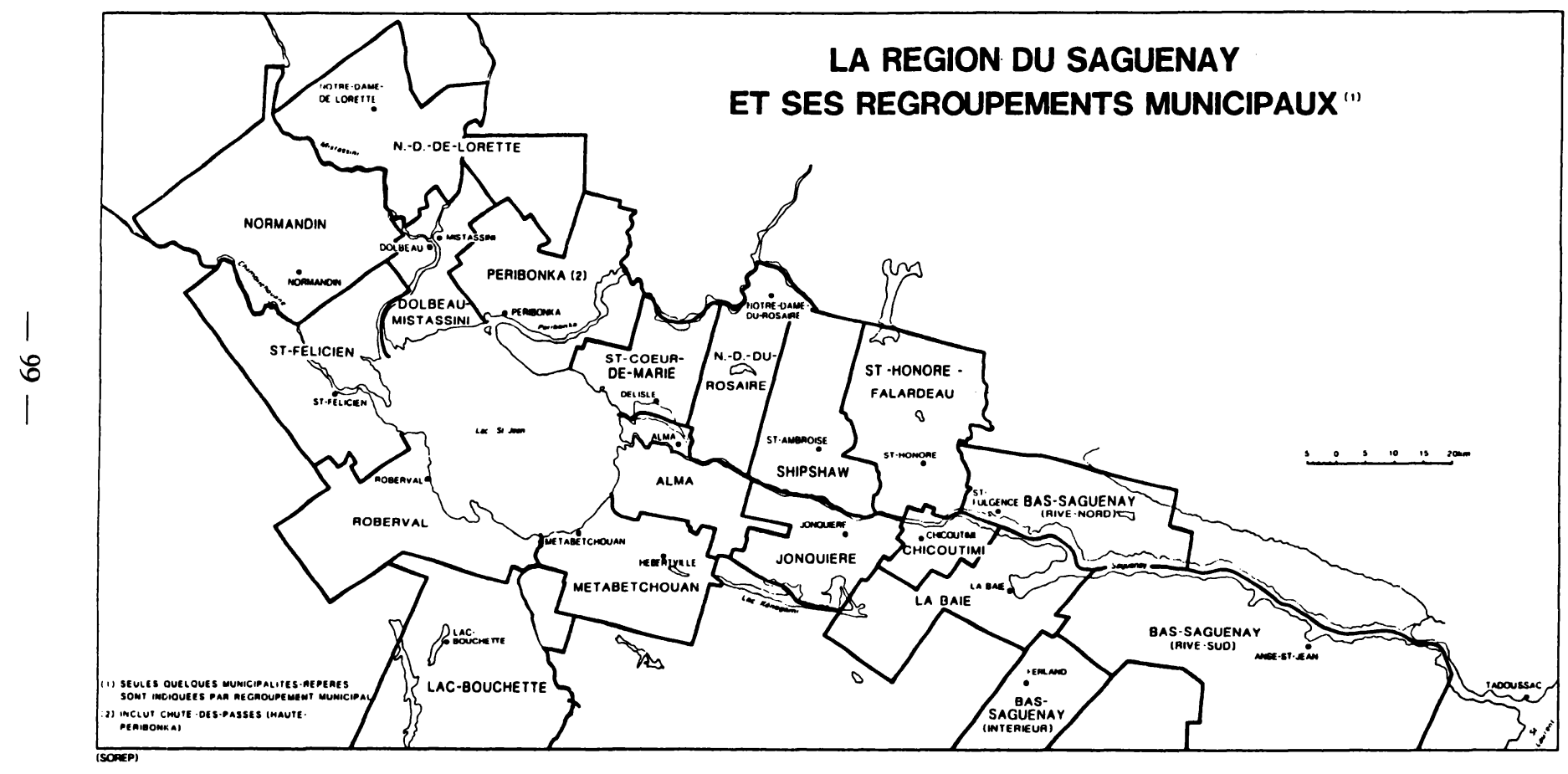




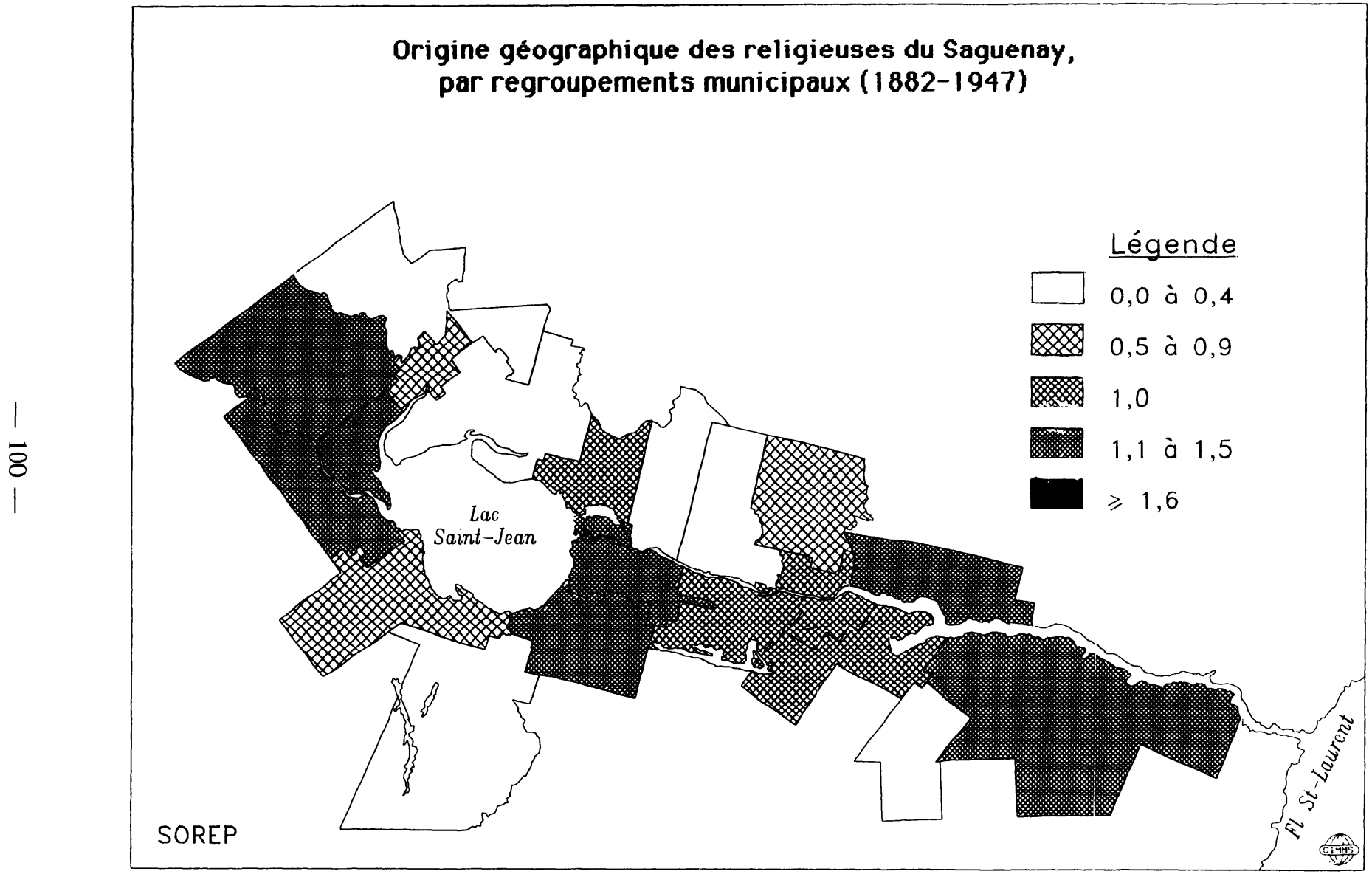


Tableau 5

Origine géographique (a) des religieuses saguenayennes, par regroupements municipaux; cinq communautés (1882-1947)

\begin{tabular}{|c|c|c|c|c|c|c|c|c|c|c|c|c|c|c|c|}
\hline \multirow[b]{2}{*}{$\begin{array}{l}\text { Regroupements } \\
\text { municipaux } \\
\text { (b) }\end{array}$} & \multicolumn{3}{|c|}{$\begin{array}{c}\text { Antoniennes de } \\
\text { Marie }\end{array}$} & \multicolumn{3}{|c|}{$\begin{array}{c}\text { Augustines } \\
\text { hospitalières }\end{array}$} & \multicolumn{3}{|c|}{ Bon-Conseil } & \multicolumn{3}{|c|}{ Bon-Pasteur } & \multicolumn{3}{|c|}{$\begin{array}{l}\text { Ursulines } \\
\text { de Roberval }\end{array}$} \\
\hline & N.a. & $\%$ & $\begin{array}{l}\text { Indice } \\
\text { comp. } \\
\text { (c) }\end{array}$ & N.a. & $\%$ & $\begin{array}{l}\text { Indice } \\
\text { comp. }\end{array}$ & N.a. & $\%$ & $\begin{array}{l}\text { Indice } \\
\text { comp. }\end{array}$ & N.a. & $\%$ & $\begin{array}{l}\text { Indice } \\
\text { comp. }\end{array}$ & N.a. & $\%$ & $\begin{array}{l}\text { Indice } \\
\text { comp. }\end{array}$ \\
\hline Bas-Sag. intérieur & 0 & 0,0 & 0,0 & 0 & 0,0 & 0,0 & 0 & 0,0 & 0,0 & 0 & 0,0 & 0,0 & 0 & 0,0 & 0,0 \\
\hline Bas Sag. rive-nord & 3 & 2,9 & 1,5 & 6 & 3,2 & 1,7 & 7 & 2,6 & 1,4 & 3 & 1,9 & 1,0 & 0 & 0,0 & 0,0 \\
\hline Bas-Sag. rive-sud & 0 & 0,0 & 0,0 & 4 & 2,2 & 0,8 & 6 & 2,2 & 0,8 & 2 & 1,3 & 0,4 & 1 & 1,9 & 1,0 \\
\hline Chicoutimi & 21 & 20,1 & 1,0 & 50 & 26,9 & 1,3 & 35 & 13,1 & 0,7 & 48 & 30,6 & 1,5 & 5 & 9,4 & 0,5 \\
\hline Jonquière & 5 & 4,8 & 0,5 & 14 & 7,6 & 0,8 & 26 & 9,7 & 1,0 & 27 & 17,2 & 1,8 & 4 & 7,6 & 0,8 \\
\hline La Baie & 11 & 10,6 & 0,9 & 30 & 16,1 & 1,3 & 21 & 7,9 & 0,7 & 36 & 23,0 & 1,9 & 6 & 11,3 & 0,9 \\
\hline St-Honoré-Falardeau & 1 & 1,0 & 1,4 & 0 & 0,0 & 0,0 & 3 & 1,1 & 1,6 & 0 & 0,0 & 0,0 & 0 & 0,0 & 0,0 \\
\hline Shipshaw & 2 & 1,9 & 0,7 & 3 & 1,6 & 0,6 & 3 & 1,1 & 0,4 & 3 & 1,9 & 0,7 & 0 & 0,0 & 0,0 \\
\hline Alma & 6 & 5,7 & 0,7 & 19 & 10,2 & 1,3 & 36 & 13,5 & 1,7 & 8 & 5,1 & 0,7 & 6 & 11,3 & 1,4 \\
\hline Métabetchouan & 23 & 22,1 & 1,8 & 22 & 11,8 & 1,0 & 47 & 17,6 & 1,4 & 13 & 8,3 & 0,7 & 15 & 28,3 & 2,3 \\
\hline N.-D. du Rosaire & 1 & 1,0 & 1,4 & 1 & 0,5 & 0,7 & 0 & 0,0 & 0,0 & 0 & 0,0 & 0,0 & 0 & 0,0 & 0,0 \\
\hline St-Cœur-de-Marie & 7 & 6,7 & 1,7 & 6 & 3,2 & 0,8 & 11 & 4,1 & 1,1 & 3 & 1,9 & 0,5 & 2 & 3,8 & 1,0 \\
\hline Dolbeau-Mistassini & 1 & 1,0 & 0,8 & 2 & 1,1 & 0,8 & 2 & 1,1 & 0,8 & 0 & 0,0 & 0,0 & 0 & 0,0 & 0,0 \\
\hline Normandin & 2 & 1,9 & 0,5 & 0 & 0,0 & 0,0 & 23 & 8,7 & 2,3 & 1 & 0,6 & 0,2 & 1 & 1,9 & 0,5 \\
\hline N.-D.-de-Lorette & 1 & 1,0 & - & 0 & 0,0 & 0,0 & 0 & 0,0 & 0,0 & 0 & 0,0 & 0,0 & 0 & 0,0 & 0,0 \\
\hline Péribonka & 1 & 1,0 & 0,8 & 0 & 0,0 & 0,0 & 3 & 1,1 & 0,8 & 0 & 0,0 & 0,0 & 0 & 0,0 & 0,0 \\
\hline Lac Bouchette & 1 & 1,0 & 0,3 & 2 & 1,1 & 0,4 & 4 & 1,5 & 0,5 & 4 & 2,5 & 0,8 & 0 & 0,0 & 0,0 \\
\hline Roberval & 13 & 12,5 & 1,4 & 7 & 3,8 & 0,4 & 17 & 6,4 & 0,7 & 4 & 2,5 & 0,3 & 8 & 15,1 & 1,7 \\
\hline St-Félicien & 5 & 4,8 & 0,6 & 20 & 10,7 & 1,3 & 22 & 8,3 & 1,0 & 5 & 3,2 & 0,4 & 5 & 9,4 & 1,2 \\
\hline Région & 104 & 100 & 1,0 & 186 & 100 & 1,0 & 266 & 100 & 1,0 & 157 & 100 & 1,0 & 53 & 100 & 1,0 \\
\hline
\end{tabular}

Notes: (a) L'origine géographique correspond au lieu de naissance. (b) Le regroupement municipal comprend quelques paroisses.

(c) L'indice comparatif est le rapport de la proportion de religieux issus d'un regroupement à la proportion de conjoints issus du même regroupement. La valeur normale est 1 .

(SOREP) 


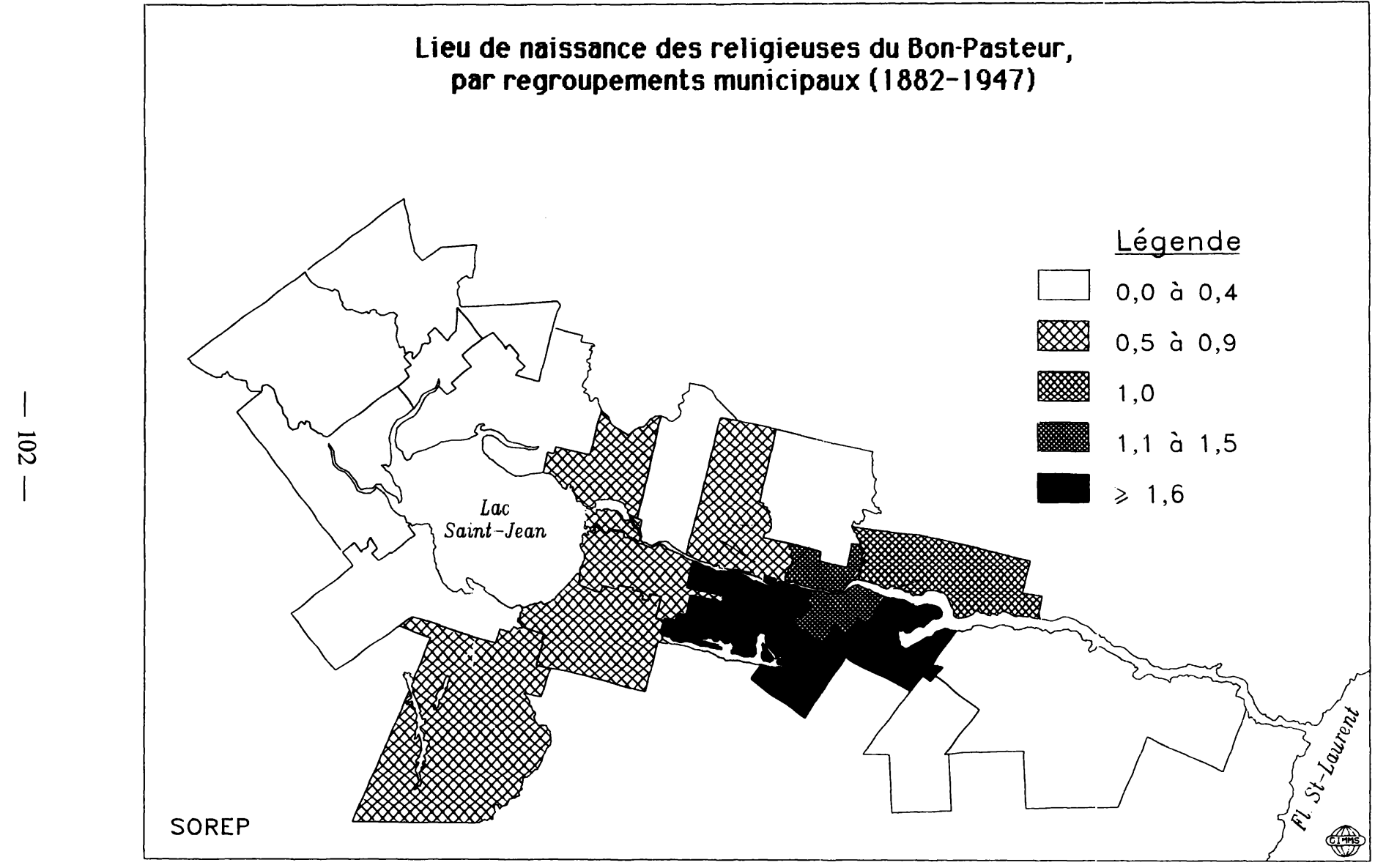




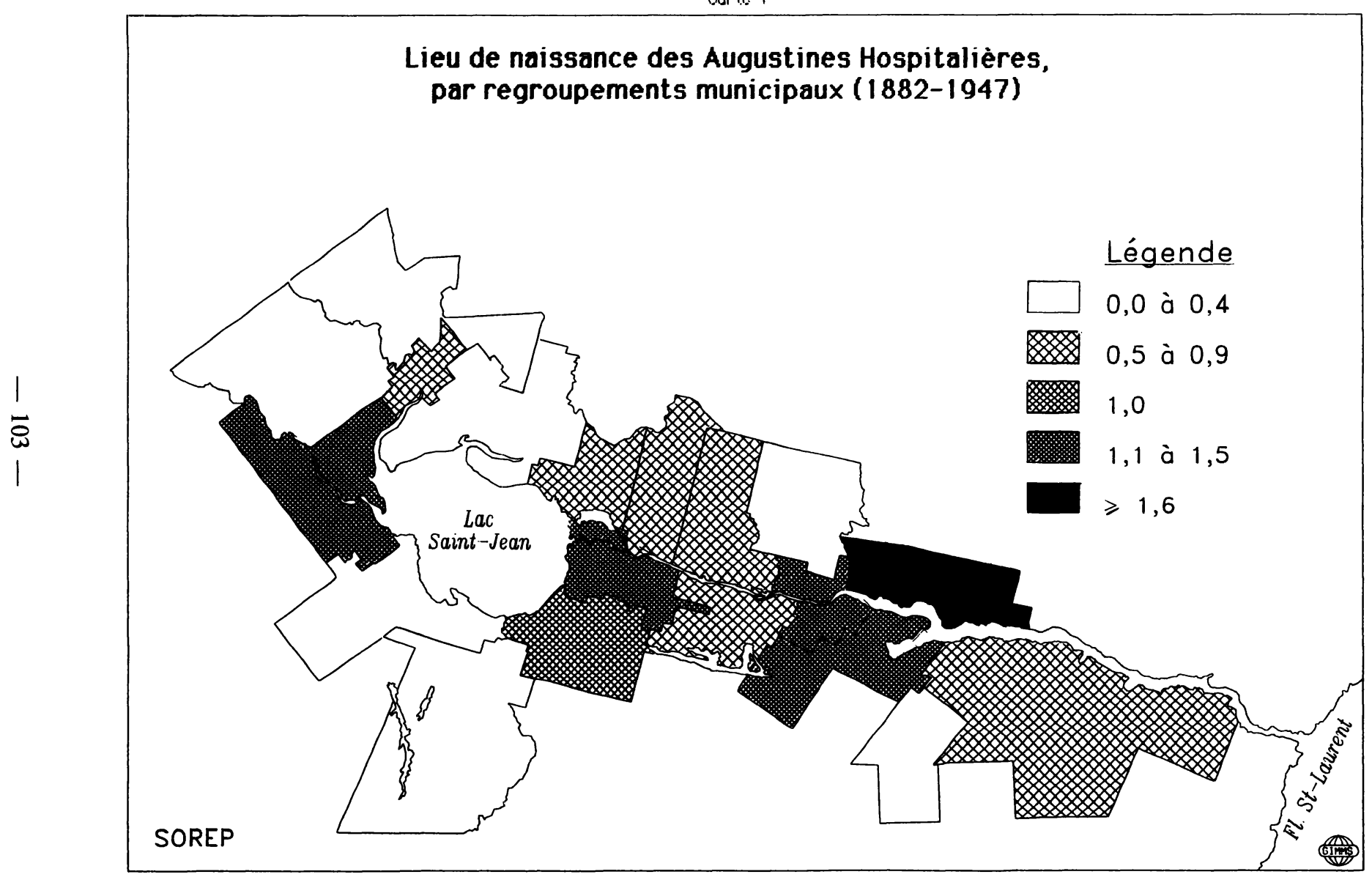




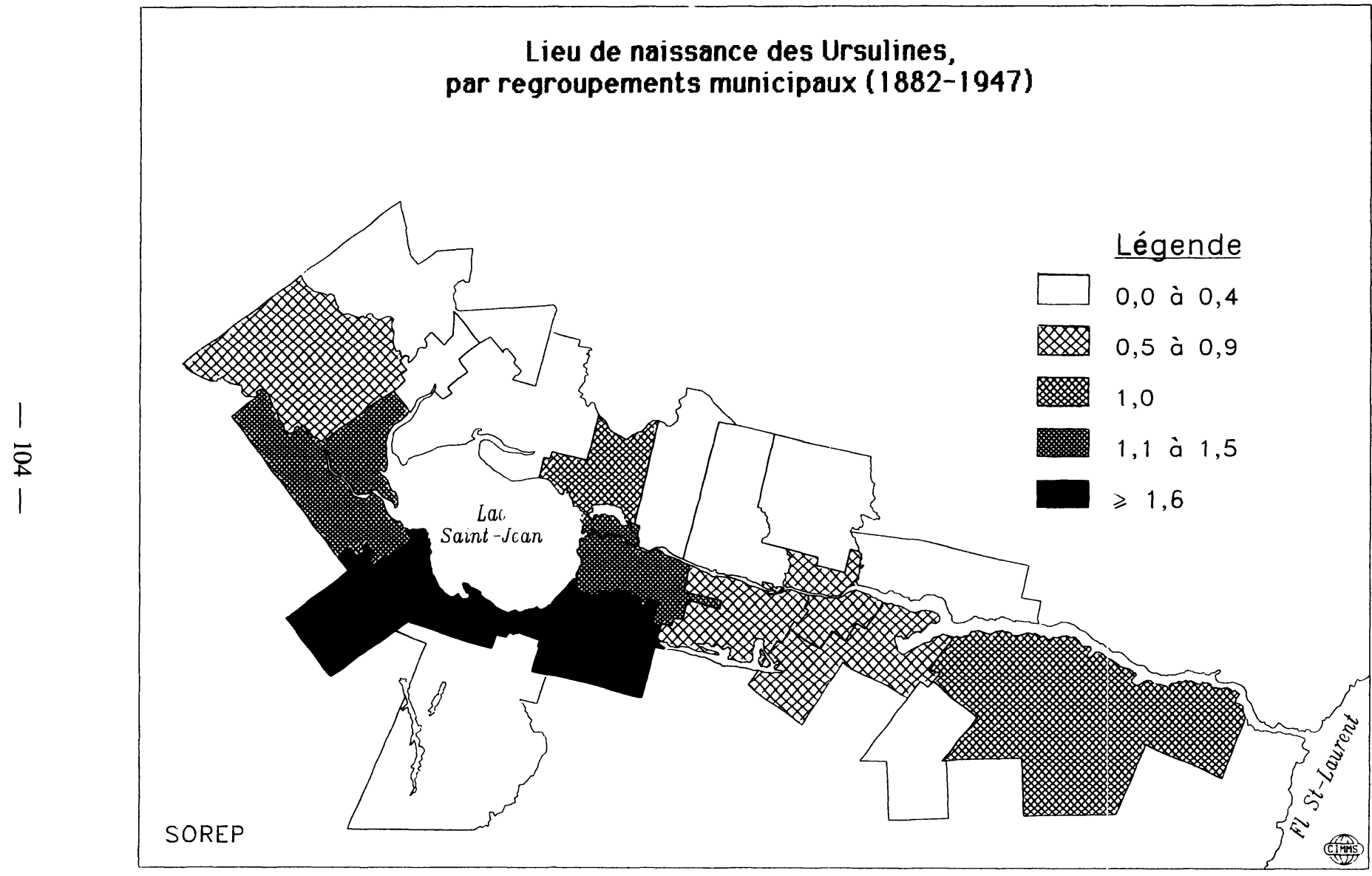




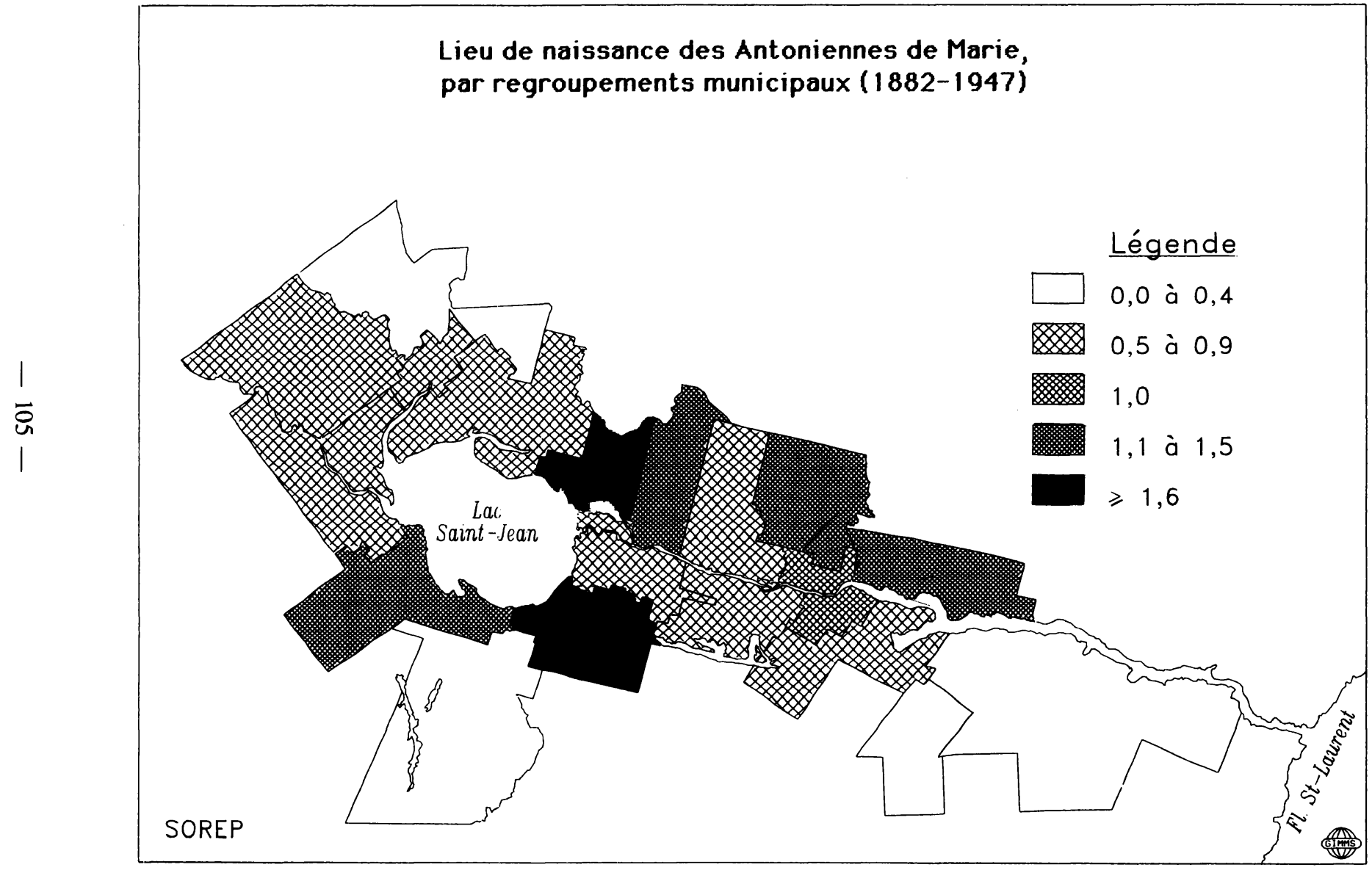




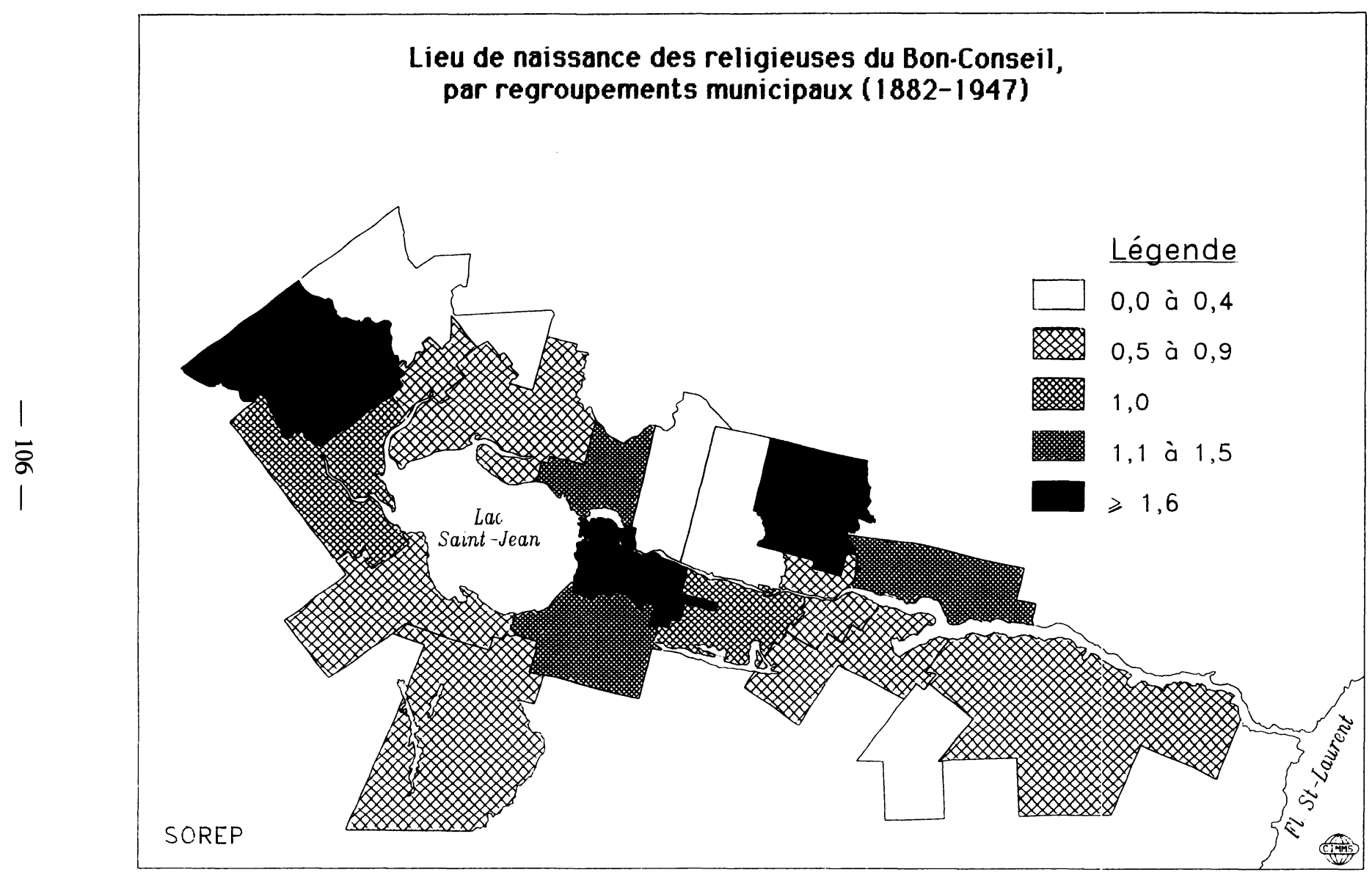


filières de recrutement appuyées sur les groupes de parenté et entretenues par le zèle de quelques religieuses.

\section{IV \\ Les origines sociales}

Prises globalement, les communautés exerçaient également un recrutement inégal, eu égard aux origines socio-professionnelles de leurs membres (tableau 6) ${ }^{10}$. Cette fois, l'indice comparatif est exprimé d'une manière un peu différente; nous produisons pour une catégorie professionnelle donnée le nombre de sujets effectivement fournis (valeur observée) et le nombre normalement attendu, étant donné le poids démographique de cette catégorie dans l'ensemble de la population. Nous éliminerons de notre commentaire les catégories comprenant des effectifs trop faibles. Pour l'ensemble de la période et pour chaque sous-période, les valeurs observées sont en excès chez les industriels et commerçants, de même que chez les artisans et gens de métier ${ }^{11}$. Par contre, les ouvriers - spécialisés ou non - affichent des valeurs déficitaires, pendant que les cultivateurs affichent une valeur observée équivalente à la valeur attendue.

Ces données suggèrent que le recrutement urbain tendait à privilégier légèrement les catégories de travailleurs non manuels. Le même phénomène survenait peut-être chez les agriculteurs. Mais pour vérifier cette hypothèse, il faudra distribuer les effectifs de cette catégorie hétéroclite en fonction de leur niveau économique et identifier les strates qui fournissaient le plus de recrues.

Par ailleurs, l'analyse par communauté révèle ici encore des clivages assez prononcés, mais ces clivages sont dans le prolongement des différenciations spatiales commentées plus haut (tableaux 7 à 11). La catégorie des cultivateurs est en excès chez les antoniennes et chez les ursulines. Pour ces dernières, c'était là un résultat prévisible puisque leur recrutement était concentré dans des zones à dominante rurale. Quant aux antoniennes en particulier, on serait tenté de relier l'orientation de leur recrutement à la nature extrêmement modeste, d'ordre domestique, de leur fonction originelle. Mais encore une fois, il faudrait d'abord s'assurer que leur recrutement faisait majoritairement appel aux couches les moins prospères du milieu agricole. En contrepartie de ce premier trait, et donc sans surprise,

10 Les catégories socio-professionnelles utilisées sont tirées d'une grille de classement mise au point par SOREP. Voir G. BOUCHARD, C. POUYEZ (1985).

11 Cette dernière catégorie regroupe des professions manuelles à caractère spécialisé, mais nous ne savons pas si leurs titulaires œuvraient à leur compte ou à salaire. Il faudrait en principe les fusionner, dans le premier cas avec les artisans, dans le second cas avec les ouvriers spécialisés. Toutefois, les valeurs obtenues dans les tableaux qui suivent, pour cette catégorie 8, donnent à penser qu'elle regroupe surtout des artisans. 
Tableau 6

Origine sociale des religieuses (a) / (Saguenay, 1882-1947)

\begin{tabular}{|c|c|c|c|c|c|c|c|c|c|c|c|c|c|c|c|c|c|c|c|c|c|c|c|c|}
\hline \multirow{3}{*}{$\begin{array}{c}\text { Catégories } \\
\text { socio- } \\
\text { professionnelles }\end{array}$} & \multicolumn{4}{|c|}{ 1882-1911 } & \multicolumn{4}{|c|}{ 1912-1921 } & \multicolumn{4}{|c|}{ 1922-1931 } & \multicolumn{4}{|c|}{ 1932-1941 } & \multicolumn{4}{|c|}{ 1942-194;" } & \multicolumn{4}{|c|}{ 1882-1947 } \\
\hline & \multicolumn{2}{|c|}{$\begin{array}{c}\text { Valeur } \\
\text { observée }\end{array}$} & \multicolumn{2}{|c|}{$\begin{array}{c}\text { Valeur } \\
\text { attendue }\end{array}$} & \multicolumn{2}{|c|}{$\begin{array}{c}\text { Valeur } \\
\text { observée }\end{array}$} & \multicolumn{2}{|c|}{$\begin{array}{c}\text { Valeur } \\
\text { attendue }\end{array}$} & \multicolumn{2}{|c|}{$\begin{array}{c}\text { Valeur } \\
\text { observée }\end{array}$} & \multicolumn{2}{|c|}{$\begin{array}{c}\text { Valeur } \\
\text { attendue }\end{array}$} & \multicolumn{2}{|c|}{$\begin{array}{c}\text { Valeur } \\
\text { observée }\end{array}$} & \multicolumn{2}{|c|}{$\begin{array}{c}\text { Valeur } \\
\text { attendue }\end{array}$} & \multicolumn{2}{|c|}{$\begin{array}{c}\text { Valeur } \\
\text { observée }\end{array}$} & \multicolumn{2}{|c|}{$\begin{array}{c}\text { Valeur } \\
\text { attendue }\end{array}$} & \multicolumn{2}{|c|}{$\begin{array}{c}\text { Valeur } \\
\text { observée }\end{array}$} & \multicolumn{2}{|c|}{$\begin{array}{c}\text { Valeur } \\
\text { attendue }\end{array}$} \\
\hline & N.a. & $\%$ & N.a. & $\%$ & N.a. & $\%$ & N.a. & $\%$ & N.a. & $\%$ & N.a. & $\%$ & N.a. & $\%$ & N.a. & $\%$ & N.a. & $\%$ & N.a. & $\%$ & N.a. & $\%$ & N.a. & $\%$ \\
\hline $\begin{array}{l}\text { 1. Administrateurs } \\
\text { et cols blancs } \\
\text { spécialisés }\end{array}$ & 1 & 0,5 & 1 & 0,4 & 1 & 0,7 & 2 & 1,2 & 7 & 2,5 & 4 & 1,3 & 4 & 1,2 & 7 & 2,2 & 3 & 2,5 & 4 & 3,4 & 16 & 1,5 & 20 & 1,8 \\
\hline $\begin{array}{l}\text { 2. Industriels et } \\
\text { commerçants }\end{array}$ & 18 & 9,1 & 5 & 2,6 & 15 & 9,7 & 7 & 4,3 & 24 & 8,4 & 13 & 4,6 & 32. & 9,9 & 15 & 4,8 & 13 & 10,7 & 6 & 5,3 & 102 & 9,4 & 48 & 4,4 \\
\hline $\begin{array}{l}\text { 3. Professions } \\
\text { libérales }\end{array}$ & 5 & 2,5 & 1 & 0,4 & 2 & 1,3 & 1 & 0,6 & 2 & 0,7 & 2 & 0,6 & 2 & 0,6 & 2 & 0,7 & 0 & 0,0 & 1 & 0,7 & 11 & 1,0 & 6 & 0,6 \\
\hline $\begin{array}{l}\text { 4. Cols blancs semi } \\
\text { et non spécialisés }\end{array}$ & 1 & 0,5 & 1 & 0,5 & 2 & 1,3 & 1 & 0,6 & 0 & 0,0 & 1 & 0,4 & 2 & 0,6 & 2 & 0,5 & 0 & 0,0 & 1 & 0,9 & 5 & 0,5 & 6 & 0,6 \\
\hline 5. Cultivateurs & 142 & 71,7 & 153 & 77,0 & 91 & 59,1 & 96 & 62,2 & 149 & 52,2 & 160 & 56,3 & 180 & 55,3 & 170 & 52,3 & 55 & 45,5 & 57 & 47,3 & 617 & 57,0 & 630 & 58,2 \\
\hline 6. Artisans & 4 & 2,0 & 2 & 1,1 & 3 & 1,9 & 2 & 1,6 & 7 & 2,5 & 4 & 1,4 & 9 & 2,8 & 6 & 1,7 & 4 & 3,3 & 2 & 1,3 & 27 & 2,5 & 15 & 1,4 \\
\hline 7. Ouvriers spécialisés & 0 & 0,0 & 0 & 0,2 & 1 & 0,7 & 0 & 0,2 & 2 & 0,7 & 2 & 0,5 & 2 & 0,6 & 5 & 1,5 & 1 & 0,8 & $?$ & 2,5 & 6 & 0,6 & 13 & 1,2 \\
\hline $\begin{array}{l}\text { 8. Gens de métier } \\
\text { (indéterminés } \\
\text { de } 6 \text { et } 7 \text { ) }\end{array}$ & 14 & 7,1 & 15 & 7,8 & 13 & 8,4 & 12 & 7,7 & 33 & 11,6 & 25 & 8,9 & 32 & 9,9 & 24 & 7,5 & 17 & 14,1 & 11 & 8,9 & 109 & 10,0 & 89 & 8.2 \\
\hline $\begin{array}{l}\text { 9. Ouvriers semi et } \\
\text { non spécialisés }\end{array}$ & 13 & 6,6 & 20 & 10,0 & 26 & 16,9 & 33 & 21,6 & 61 & 21,4 & 74 & 26,0 & 62 & 19,1 & 94 & 28,8 & 28 & 23,1 & 36 & 29,7 & 190 & 17,5 & 256 & 23,6 \\
\hline Toutes catégories (b) & 198 & 100 & 198 & 100 & 154 & 100 & 154 & 100 & 285 & 100 & 285 & 100 & 325 & 100 & 325 & 100 & 121 & 100 & 121 & 100 & 1083 & 100 & 1083 & 100 \\
\hline
\end{tabular}

Notes: (a) Dans ce tableau, les valeurs attendues sont celles que l'on devrait obtenir si la distribution socio-professionnelle des religieuses était proportionnelle aux effectifs de chaque catégorie dans l'ensemble de la population. Ces effectifs ont été établis à partir d'un groupe-témoin formé des 19719 femmes nées et ayant contracté un premier mariage au Saguenay entre 1882-1947.

(b) Nombre de professions indéterminées: 35. 
on relève chez ces deux communautés un déficit dans la catégorie des ouvriers semi et non spécialisés ${ }^{12}$. C'est chez le Bon-Conseil et le BonPasteur que cette catégorie est la plus représentée. Dans le cas du BonPasteur, le fait est intéressant puisqu'on y trouve également une forte représentation des artisans et gens de métier, soit la couche supérieure des travailleurs manuels. Nous pensons que ces données en apparence paradoxales reflètent un recrutement à dominante urbaine plus qu'une sélection sur une base socio-économique.

Les quelques données comparatives dont il est possible de faire état ici se refèrent toutes à la Nouvelle-France. Ce terme de comparaison est assurément éloigné du contexte de notre étude; nous nous y référons néanmoins, faute de mieux. Les données disponibles établissent que, contrairement à ce qui se passait au Saguenay à une époque beaucoup plus récente, les religieuses provenaient massivement des milieux favorisés (familles de nobles, de marchands, d'officiers de l'armée) (L. PELLETIER, 1986, tableau 4.17). Par ailleurs, d'après l'enquête de M. DALLAIRE (1986, chap. VI), cette orientation du recrutement se serait atténuée dans la deuxième moitié du XVIIIe siècle. Mais ce dernier résultat est sujet à caution, puisque l'auteure - elle le reconnaît elle-même - n'a pas pu rapporter les effectifs de religieuses à leur population de base. La même enquête (chap. 6) révèle aussi l'existence d'une stratification socioéconomique entre les communautés; on retrouve ici le phénomène important de l'hétérogénéité, déjà évoqué à propos du Saguenay et relevé également par M. DANYLEWYCZ (1987, chap. 3) pour la période 1890-1920. Encore une fois cependant, dans cette dernière enquête, les statistiques présentées ne sont pas pondérées par une référence à la population de base (ex.: tableaux 12 et 14); mais selon toute vraisemblance, l'orientation du recrutement variait sensiblement entre les communautés étudiées, surtout entre la Congrégation de Notre-Dame (très élitiste) et les sœurs de la Miséricorde (à forte représentation ouvrière et paysanne).

\section{$\mathbf{V}$ \\ Conclusion}

Ce sont là les principaux résultats d'une enquête qui en est à ses premières étapes, en ce qui concerne l'analyse comme telle. À ce stade-ci, elle attire surtout l'attention sur les points suivants:

12 Notons que nous parlons ici d'excès ou de déficit par référence à la norme dégagée pour l'ensemble des cinq communautés. Ainsi, les ouvriers semi et non spécialisés sont sousreprésentés dans toutes les communautés, mais ils le sont davantage chez les ursulines et les antoniennes. 
Tableau 7

Origine sociale des antoniennes de Marie (a) / (Saguenay, 1882-1947)

\begin{tabular}{|c|c|c|c|c|c|c|c|c|c|c|c|c|c|c|c|c|c|c|c|c|}
\hline \multirow{3}{*}{$\begin{array}{c}\text { Catégories } \\
\text { socio- } \\
\text { professionnelles }\end{array}$} & \multicolumn{4}{|c|}{ 1882-1911 } & \multicolumn{4}{|c|}{ 1912-1921 } & \multicolumn{4}{|c|}{$1922-1931$} & \multicolumn{4}{|c|}{$1932-1941$} & \multicolumn{4}{|c|}{$1942-1947$} \\
\hline & \multicolumn{2}{|c|}{$\begin{array}{c}\text { Valeur } \\
\text { observée }\end{array}$} & \multicolumn{2}{|c|}{$\begin{array}{c}\text { Valeur } \\
\text { attendue }\end{array}$} & \multicolumn{2}{|c|}{$\begin{array}{c}\text { Valeur } \\
\text { observée }\end{array}$} & \multicolumn{2}{|c|}{$\begin{array}{c}\text { Valeur } \\
\text { attendue }\end{array}$} & \multicolumn{2}{|c|}{$\begin{array}{c}\text { Valeur } \\
\text { observée }\end{array}$} & \multicolumn{2}{|c|}{$\begin{array}{c}\text { Valeur } \\
\text { attendue }\end{array}$} & \multicolumn{2}{|c|}{$\begin{array}{c}\text { Valeur } \\
\text { observée }\end{array}$} & \multicolumn{2}{|c|}{$\begin{array}{c}\text { Valeur } \\
\text { attendue }\end{array}$} & \multicolumn{2}{|c|}{$\begin{array}{c}\text { Valeur } \\
\text { observée }\end{array}$} & \multicolumn{2}{|c|}{$\begin{array}{c}\text { Valeur } \\
\text { attendue }\end{array}$} \\
\hline & N.a. & $\%$ & N.a. & $\%$ & N.a. & $\%$ & N.a. & $\%$ & N.a. & $\%$ & N.a. & $\%$ & N.a. & $\%$ & N.a. & $\%$ & N.a. & $\%$ & N.a. & $\%$ \\
\hline $\begin{array}{l}\text { 1. Administrateurs } \\
\text { et cols blancs } \\
\text { spécialisés }\end{array}$ & 0 & 0,0 & 0 & 0,4 & 0 & 0,0 & 0 & 1,2 & 0 & 0,0 & 0 & 1,3 & 0 & 0,0 & 1 & 2,2 & 0 & 0,0 & 0 & 3,4 \\
\hline $\begin{array}{l}\text { 2. Industriels et } \\
\text { commerçants }\end{array}$ & 1 & 8,3 & 0 & 2,6 & 2 & 10,0 & 1 & 4,3 & 2 & 9,5 & 1 & 4,6 & 1 & 3,0 & 2 & 4,8 & 2 & 15,4 & 1 & 5,3 \\
\hline $\begin{array}{l}\text { 3. Professions } \\
\text { libérales }\end{array}$ & 0 & 0,0 & 0 & 0,4 & 0 & 0,0 & 0 & 0,6 & 0 & 0,0 & 0 & 0,6 & 1 & 3,0 & 0 & 0,7 & 0 & 0,0 & 0 & 0,7 \\
\hline $\begin{array}{l}\text { 4. Cols blancs semi } \\
\text { et non spécialisés }\end{array}$ & 0 & 0,0 & 0 & 0,5 & 1 & 5,0 & 0 & 0,6 & 0 & 0,0 & 0 & 0,4 & 0 & 0,0 & 0 & 0,5 & 0 & 0,0 & 0 & 0,9 \\
\hline 5. Cultivateurs & 10 & 83,4 & 9 & 77,0 & 11 & 55,0 & 13 & 62,2 & 17 & 80,9 & 12 & 56,3 & 24 & 72,8 & 17 & 52,3 & 10 & 76,9 & 6 & 47,3 \\
\hline 6. Artisans & 0 & 0,0 & 0 & 1,1 & 0 & 0,0 & 0 & 1,6 & 0 & 0,0 & 0 & 1,4 & 1 & 3,0 & 1 & 1,7 & 0 & 0,0 & 0 & 1,3 \\
\hline 7. Ouvriers spécialisés & 0 & 0,0 & 0 & 0,2 & 0 & 0,0 & 0 & 0,2 & 0 & 0,0 & 0 & 0,5 & 0 & 0,0 & 1 & 1,5 & 0 & 0,0 & 0 & 2,5 \\
\hline $\begin{array}{l}\text { 8. Gens de métier } \\
\text { (indéterminés } \\
\text { de } 6 \text { et } 7 \text { ) }\end{array}$ & 0 & 0,0 & 1 & 7,8 & 3 & 15,0 & 2 & 7,7 & 1 & 4,8 & 2 & 8,9 & 3 & 9,1 & 2 & 7,5 & 0 & 0,0 & 2 & 8.9 \\
\hline $\begin{array}{l}\text { 9. Ouvriers semi et } \\
\text { non spécialisés }\end{array}$ & 1 & 8,3 & 2 & 10,0 & 3 & 15,0 & 4 & 21,6 & 1 & 4,8 & 6 & 26,0 & 3 & 9,1 & 9 & 28,8 & 1 & 7,7 & 4 & 29.7 \\
\hline Toutes catégories (b) & 12 & 100 & 12 & 100 & 20 & 100 & 20 & 100 & 21 & 100 & 21 & 100 & 33 & 100 & 33 & 100 & 13 & 100 & 13 & 100 \\
\hline
\end{tabular}

\begin{tabular}{|c|c|c|c|}
\hline \multicolumn{4}{|c|}{ 1882-1947 } \\
\hline \multicolumn{2}{|c|}{$\begin{array}{c}\text { Valeur } \\
\text { observée }\end{array}$} & \multicolumn{2}{|c|}{$\begin{array}{c}\text { Valeur } \\
\text { attendue }\end{array}$} \\
\hline N.a. & $\%$ & N.a. & $\%$ \\
\hline 0 & 0,0 & 2 & 1,8 \\
\hline 8 & 8,1 & 4 & 4,4 \\
\hline 1 & 1,0 & 1 & 0,6 \\
\hline 1 & 1,0 & 1 & 0,6 \\
\hline 72 & 72,7 & 58 & 58,2 \\
\hline 1 & 1,0 & 1 & 1,4 \\
\hline 0 & 0,0 & 1 & 1,2 \\
\hline 7 & 7,1 & 8 & 8,2 \\
\hline 9 & 9,1 & 23 & 23,6 \\
\hline 99 & 100 & 99 & 100 \\
\hline
\end{tabular}

Notes: (a) Dans ce tableau, les valeurs attendues sont celles que l'on devrait obtenir si la distribution socio-professionnelle des antoniennes de Marie était proportionnelle aux effectifs de chaque catégorie dans l'ensemble de la population. C'es effectifs ont été établis à partir d'un groupe-témoin formé des 19719 femmes nées et ayant contracté un premier mariage au Saguenay entre 1882 et 1947.

(b) Nombre de professions indéterminées: 5.

(SOREP) 
Tableau 8

Origine sociale des ursulines de Roberval (a) / (Saguenay, 1882-1941)

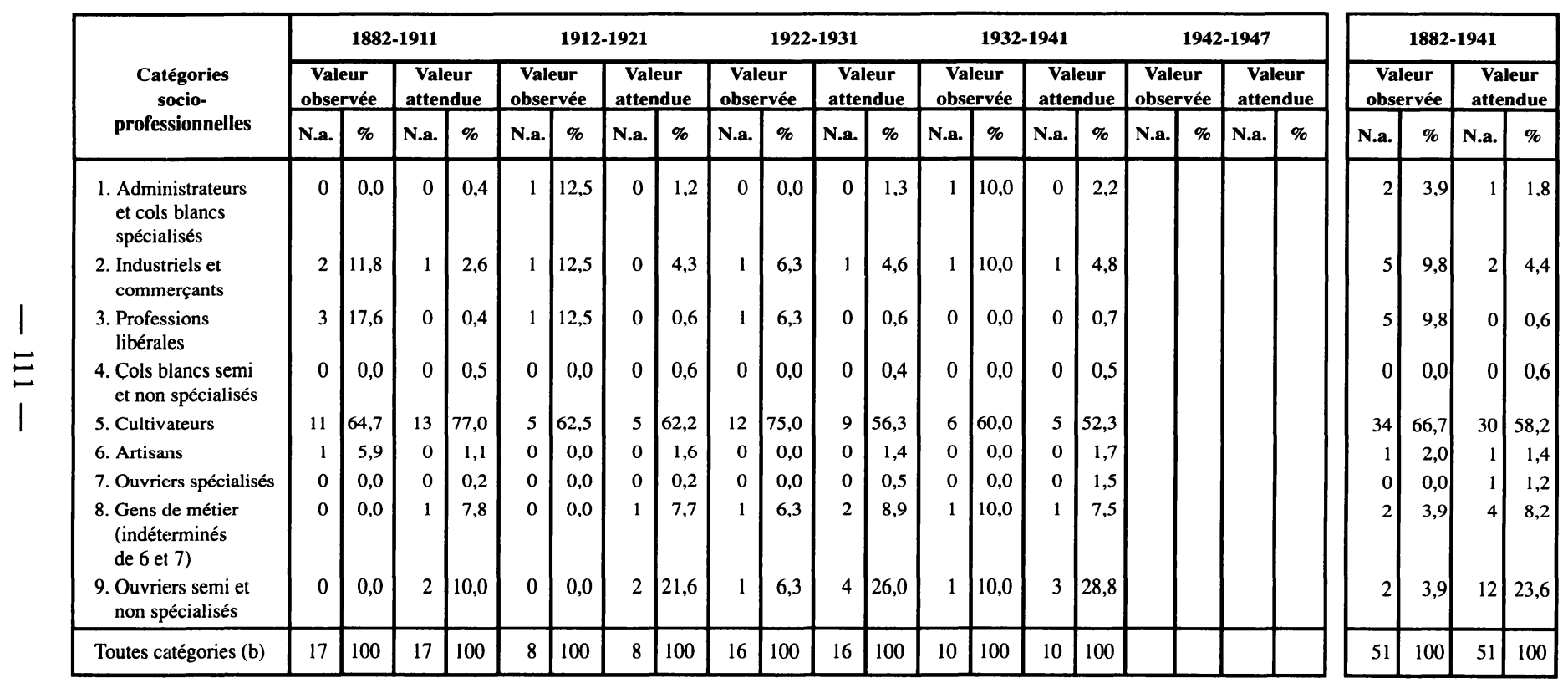

Notes: (a) Dans ce tableau, les valeurs attendues sont celles que l'on devrait obtenir si la distribution socio-professionnelle des ursulines était proportionnelle aux effectifs de chaque catégorie dans l'ensemble de la population. Ces effectifs ont été établis à partir d'un groupe-témoin formé des 19719 femmes nées et ayant contracté un premier mariage au Saguenay entre 1882 et 1947.

(b) Nombre de professions indéterminées: 2.

(SOREP) 
Tableau 9

Origine sociale des Sœurs du Bon-Conseil (a) / (Saguenay, 1882-1947)

\begin{tabular}{|c|c|c|c|c|c|c|c|c|c|c|c|c|c|c|c|c|c|c|c|c|c|c|c|c|}
\hline \multirow{3}{*}{$\begin{array}{c}\text { Catégories } \\
\text { socio- } \\
\text { professionnelles }\end{array}$} & \multicolumn{4}{|c|}{ 1882-1911 } & \multicolumn{4}{|c|}{ 1912-1921 } & \multicolumn{4}{|c|}{$1922-1931$} & \multicolumn{4}{|c|}{ 1932-1941 } & \multicolumn{4}{|c|}{$1942-19<47$} & \multicolumn{4}{|c|}{ 1882-1947 } \\
\hline & \multicolumn{2}{|c|}{\begin{tabular}{|c|}
$\begin{array}{c}\text { Valeur } \\
\text { observée }\end{array}$ \\
\end{tabular}} & \multicolumn{2}{|c|}{$\begin{array}{c}\begin{array}{c}\text { Valeur } \\
\text { attendue }\end{array} \\
\end{array}$} & \multicolumn{2}{|c|}{$\begin{array}{c}\text { Valeur } \\
\text { observée }\end{array}$} & \multicolumn{2}{|c|}{\begin{tabular}{|c|} 
Valeur \\
attendue
\end{tabular}} & \multicolumn{2}{|c|}{\begin{tabular}{|c|}
$\begin{array}{c}\text { Valeur } \\
\text { observée }\end{array}$ \\
\end{tabular}} & \multicolumn{2}{|c|}{$\begin{array}{c}\text { Valeur } \\
\text { attendue }\end{array}$} & \multicolumn{2}{|c|}{$\begin{array}{c}\begin{array}{c}\text { Valeur } \\
\text { observée }\end{array} \\
\end{array}$} & \multicolumn{2}{|c|}{$\begin{array}{c}\text { Valeur } \\
\text { attendue }\end{array}$} & \multicolumn{2}{|c|}{\begin{tabular}{|c|}
$\begin{array}{c}\text { Valeur } \\
\text { observée }\end{array}$ \\
\end{tabular}} & \multicolumn{2}{|c|}{\begin{tabular}{|c|} 
Valeur \\
attendue
\end{tabular}} & \multicolumn{2}{|c|}{$\begin{array}{c}\text { Valeur } \\
\text { observée }\end{array}$} & \multicolumn{2}{|c|}{$\begin{array}{c}\begin{array}{c}\text { Valeur } \\
\text { attendue }\end{array} \\
\end{array}$} \\
\hline & N.a. & $\%$ & N.a. & $\%$ & N.a. & $\%$ & N.a. & $\%$ & N.a. & $\%$ & N.a. & $\%$ & N.a. & $\%$ & N.a. & $\%$ & N.a. & $\%$ & N.a. & $\%$ & N.a. & $\%$ & N.a. & $\%$ \\
\hline $\begin{array}{l}\text { 1. Administrateurs } \\
\text { et cols blancs } \\
\text { spécialisés }\end{array}$ & 0 & 0,0 & 0 & 0,4 & 0 & 0,0 & 0 & 1,2 & 3 & 4,7 & 1 & 1,3 & 1 & 1,1 & 2 & 2,2 & 0 & 0,0 & 1 & 3,4 & 4 & 1,5 & 5 & 1,8 \\
\hline $\begin{array}{l}\text { 2. Industriels et } \\
\text { commerçants }\end{array}$ & 5 & 12,8 & 1 & 2,6 & 7 & 20,6 & 2 & 4,3 & 5 & 7,8 & 3 & 4,6 & 3 & 3,5 & 4 & 4,8 & 2 & 5,7 & 2 & 5,3 & 22 & 8,5 & 11 & 4,4 \\
\hline $\begin{array}{l}\text { 3. Professions } \\
\text { libérales }\end{array}$ & 0 & 0,0 & 0 & 0,4 & 0 & 0,0 & 0 & 0,6 & 0 & 0,0 & 0 & 0,6 & 1 & 1,1 & 1 & 0,7 & 0 & 0,0 & (1 & 0,7 & 1 & 0,4 & 2 & 0,6 \\
\hline $\begin{array}{l}\text { 4. Cols blancs semi } \\
\text { et non spécialisés }\end{array}$ & 0 & 0,0 & 0 & 0,5 & 0 & 0,0 & 0 & 0,6 & 0 & 0,0 & 0 & 0,4 & 0 & 0,0 & 0 & 0,5 & 0 & 0,0 & (1 & 0,9 & 0 & 0,0 & 2 & 0,6 \\
\hline 5. Cultivateurs & 29 & 74,3 & 30 & 77,0 & 18 & 52,9 & 21 & 62,2 & 33 & 51,5 & 36 & 56,3 & 55 & 63,3 & 46 & 52,3 & 12 & 34,3 & $1 \%$ & 47,3 & 147 & 56,8 & 151 & 58,2 \\
\hline 6. Artisans & 1 & 2,6 & 1 & 1,1 & 0 & 0,0 & 1 & 1,6 & 1 & 1,6 & 1 & 1,4 & 4 & 4,6 & 1 & 1,7 & 0 & 0,0 & 0 & 1,3 & 6 & 2,3 & 3 & 1,4 \\
\hline 7. Ouvriers spécialisés & 0 & 0,0 & 0 & 0,2 & 0 & 0,0 & 0 & 0,2 & 1 & 1,6 & 0 & 0,5 & 1 & 1,1 & 1 & 1,5 & 0 & 0,0 & 1 & 2,5 & 2 & 0,8 & 3 & 1,2 \\
\hline $\begin{array}{l}\text { 8. Gens de métier } \\
\text { (indéterminés } \\
\text { de } 6 \text { et } 7 \text { ) }\end{array}$ & 4 & 10,3 & 3 & 7,8 & 4 & 11,8 & 3 & 7,7 & 8 & 12,5 & 6 & 8,9 & 6 & 6,9 & 7 & 7,5 & 8 & 22,9 & 3 & 8,9 & 30 & 11,6 & 21 & 8,2 \\
\hline $\begin{array}{l}\text { 9. Ouvriers semi et } \\
\text { non spécialisés }\end{array}$ & 0 & 0,0 & 4 & 10,0 & 5 & 14,7 & 7 & 21,6 & 13 & 20,3 & 17 & 26,0 & 16 & 18,4 & 25 & 28,8 & 13 & 37,1 & 11 & 29,7 & 47 & 18,1 & 61 & 23,6 \\
\hline Toutes catégories (b) & 39 & 100 & 39 & 100 & 34 & 100 & 34 & 100 & 64 & 100 & 64 & 100 & 87 & 100 & 87 & 100 & 35 & 100 & 35 & 100 & 259 & 100 & 259 & 100 \\
\hline
\end{tabular}

Notes: (a) Dans ce tableau, les valeurs attendues sont celles que l'on devrait obtenir si la distribution socio-professionnelle des Sœurs était proportionnelle aux effectifs de chaque catégorie dans l'ensemble de la population. Ces effectifs ont été établis à partir d'un groupe-témoin formé des 19719 femmes nées et ayant contracté un premier mariage au Saguenay entre 1882 et 1947.

(b) Nombre de professions indéterminées: 7. 
Tableau 10

Origine sociale des Sœurs du Bon-Pasteur (a) / (Saguenay, 1882-1947)

\begin{tabular}{|c|c|c|c|c|c|c|c|c|c|c|c|c|c|c|c|c|c|c|c|c|c|c|c|c|}
\hline \multirow{3}{*}{$\begin{array}{c}\text { Catégories } \\
\text { socio- } \\
\text { professionnelles }\end{array}$} & \multicolumn{4}{|c|}{ 1882-1911 } & \multicolumn{4}{|c|}{ 1912-1921 } & \multicolumn{4}{|c|}{ 1922-1931 } & \multicolumn{4}{|c|}{ 1932-1941 } & \multicolumn{4}{|c|}{ 1942-1947 } & \multicolumn{4}{|c|}{ 1882-1947 } \\
\hline & \multicolumn{2}{|c|}{$\begin{array}{c}\text { Valeur } \\
\text { observée }\end{array}$} & \multicolumn{2}{|c|}{\begin{tabular}{|c|} 
Valeur \\
attendue
\end{tabular}} & \multicolumn{2}{|c|}{\begin{tabular}{|c|}
$\begin{array}{c}\text { Valeur } \\
\text { observée }\end{array}$ \\
\end{tabular}} & \multicolumn{2}{|c|}{$\begin{array}{c}\text { Valeur } \\
\text { attendue }\end{array}$} & \multicolumn{2}{|c|}{\begin{tabular}{|c|}
$\begin{array}{c}\text { Valeur } \\
\text { observée }\end{array}$ \\
\end{tabular}} & \multicolumn{2}{|c|}{$\begin{array}{l}\text { Valeur } \\
\text { attendue }\end{array}$} & \multicolumn{2}{|c|}{$\begin{array}{c}\begin{array}{c}\text { Valeur } \\
\text { observée }\end{array} \\
\end{array}$} & \multicolumn{2}{|c|}{$\begin{array}{c}\text { Valeur } \\
\text { attendue }\end{array}$} & \multicolumn{2}{|c|}{\begin{tabular}{|c|}
$\begin{array}{c}\text { Valeur } \\
\text { observée }\end{array}$ \\
\end{tabular}} & \multicolumn{2}{|c|}{\begin{tabular}{|c|} 
Valeur \\
attendue
\end{tabular}} & \multicolumn{2}{|c|}{$\begin{array}{c}\text { Valeur } \\
\text { observée }\end{array}$} & \multicolumn{2}{|c|}{$\begin{array}{c}\text { Valeur } \\
\text { attendue }\end{array}$} \\
\hline & N.a. & $\%$ & N.a. & $\%$ & N.a. & $\%$ & N.a. & $\%$ & N.a. & $\%$ & N.a. & $\%$ & N.a. & $\%$ & N.a. & $\%$ & N.a. & $\%$ & N.a. & $\%$ & N.a. & $\%$ & N.a. & $\%$ \\
\hline $\begin{array}{l}\text { 1. Administrateurs } \\
\text { et cols blancs } \\
\text { spécialisés }\end{array}$ & 1 & 7,1 & 0 & 0,4 & 0 & 0,0 & 0 & 1,2 & 1 & 2,2 & 1 & 1,3 & 2 & 4,1 & 1 & 2,2 & 1 & 7,1 & 0 & 3,4 & 5 & 3,9 & 2 & 1,8 \\
\hline $\begin{array}{l}\text { 2. Industriels et } \\
\text { commerçants }\end{array}$ & 3 & 21,5 & 0 & 2,6 & 1 & 12,5 & 0 & 4,3 & 1 & 2,2 & 2 & 4,6 & 2 & 4,1 & 2 & 4,8 & 1 & 7,1 & 1 & 5,3 & 8 & 6,1 & 6 & 4,4 \\
\hline $\begin{array}{l}\text { 4. Cols blancs semi } \\
\text { et non spécialisés }\end{array}$ & 0 & 0,0 & 0 & 0,5 & 0 & 0,0 & 0 & 0,6 & 0 & 0,0 & 0 & 0,4 & 2 & 4,1 & 0 & 0,5 & 0 & 0,0 & 0 & 0,9 & 2 & 1,5 & 1 & 0,6 \\
\hline 5. Cultivateurs & 9 & 64,3 & 11 & 77,0 & 2 & 25,0 & 5 & 62,2 & 21 & 46,7 & 25 & 56,3 & 25 & 51,0 & 26 & 52,3 & 4 & 28,6 & 7 & 47,3 & 61 & 46,9 & 75 & 58,2 \\
\hline 6. Artisans & 0 & 0,0 & 0 & 1,1 & 0 & 0,0 & 0 & 1,6 & 3 & 6,7 & 1 & 1,4 & 1 & 2,0 & 1 & 1,7 & 0 & 0,0 & 0 & 1,3 & 4 & 3,1 & 2 & 1,4 \\
\hline 7. Ouvriers spécialisés & 0 & 0,0 & 0 & 0,2 & 0 & 0,0 & 0 & 0,2 & 2 & 4,4 & 0 & 0,5 & 1 & 2,0 & 1 & 1,5 & 0 & 0,0 & 0 & 2,5 & 3 & 2,3 & 2 & 1,2 \\
\hline $\begin{array}{l}\text { 8. Gens de métier } \\
\text { (indéterminés } \\
\text { de } 6 \text { et } 7 \text { ) }\end{array}$ & 0 & 0,0 & 1 & 7,8 & 2 & 25,0 & 1 & 7,7 & 8 & 17,8 & 4 & 8,9 & 5 & 10,2 & 4 & 7,5 & 5 & 35,7 & 2 & 8,9 & 20 & 15,4 & 11 & 8,2 \\
\hline Toutes catégories (b) & 14 & 100 & 14 & 100 & 8 & 100 & 8 & 100 & 45 & 100 & 45 & 100 & 49 & 100 & 49 & 100 & 14 & 100 & 14 & 100 & 130 & 100 & 130 & 100 \\
\hline
\end{tabular}

Notes: (a) Dans ce tableau, les valeurs attendues sont celles que l'on devrait obtenir si la distribution socio-professionnelle des sœurs était proportionnelle aux effectifs de chaque catégorie dans l'ensemble de la population. Ces effectifs ont été établis à partir d'un groupe-témoin formé des 19719 femmes nées et ayant contracté un premier mariage au Saguenay entre 1882 et 1947.

(b) Nombre de professions indéterminées: 9.

(SOREP) 
Tableau 11

Origine sociale des augustines hospitalières (a) / (Saguenay, 1882-1947)

\begin{tabular}{|c|c|c|c|c|c|c|c|c|c|c|c|c|c|c|c|c|c|c|c|c|c|c|c|c|}
\hline \multirow{3}{*}{$\begin{array}{c}\text { Catégories } \\
\text { socio- } \\
\text { professionnelles }\end{array}$} & \multicolumn{4}{|c|}{ 1882-1911 } & \multicolumn{4}{|c|}{ 1912-1921 } & \multicolumn{4}{|c|}{$1922-1931$} & \multicolumn{4}{|c|}{ 1932-1941 } & \multicolumn{4}{|c|}{$1942-1947$} & \multicolumn{4}{|c|}{ 1882-1947 } \\
\hline & \multicolumn{2}{|c|}{\begin{tabular}{|c|}
$\begin{array}{c}\text { Valeur } \\
\text { observée }\end{array}$ \\
\end{tabular}} & \multicolumn{2}{|c|}{\begin{tabular}{|c|c}
$\begin{array}{c}\text { Valeur } \\
\text { attendue }\end{array}$ \\
\end{tabular}} & \multicolumn{2}{|c|}{\begin{tabular}{|c|}
$\begin{array}{c}\text { Valeur } \\
\text { observée }\end{array}$ \\
\end{tabular}} & \multicolumn{2}{|c|}{\begin{tabular}{|c|}
$\begin{array}{c}\text { Valeur } \\
\text { attendue }\end{array}$ \\
\end{tabular}} & \multicolumn{2}{|c|}{\begin{tabular}{|c|}
$\begin{array}{c}\text { Valeur } \\
\text { observée }\end{array}$ \\
\end{tabular}} & \multicolumn{2}{|c|}{\begin{tabular}{|c|}
$\begin{array}{c}\text { Valeur } \\
\text { attendue }\end{array}$ \\
\end{tabular}} & \multicolumn{2}{|c|}{$\begin{array}{c}\text { Valeur } \\
\text { observée }\end{array}$} & \multicolumn{2}{|c|}{\begin{tabular}{|c|}
$\begin{array}{c}\text { Valeur } \\
\text { attendue }\end{array}$ \\
\end{tabular}} & \multicolumn{2}{|c|}{\begin{tabular}{|c|}
$\begin{array}{c}\text { Valeur } \\
\text { observée }\end{array}$ \\
\end{tabular}} & \multicolumn{2}{|c|}{$\begin{array}{c}\begin{array}{c}\text { Valeur } \\
\text { attendue }\end{array} \\
\end{array}$} & \multicolumn{2}{|c|}{\begin{tabular}{|c|}
$\begin{array}{c}\text { Valeur } \\
\text { observée }\end{array}$ \\
\end{tabular}} & \multicolumn{2}{|c|}{$\begin{array}{c}\text { Valeur } \\
\text { attendue }\end{array}$} \\
\hline & N.a. & $\%$ & N.a. & $\%$ & N.a. & $\%$ & N.a. & $\%$ & N.a. & $\%$ & N.a. & $\%$ & N.a. & $\%$ & N.a. & $\%$ & N.a. & $\%$ & N.a. & $\%$ & N.a. & $\%$ & N.a. & $\%$ \\
\hline $\begin{array}{l}\text { 1. Administrateurs } \\
\text { et cols blancs } \\
\text { spécialisés }\end{array}$ & 0 & 0,0 & 0 & 0,4 & 0 & 0,0 & 0 & 1,2 & 0 & 0,0 & 1 & 1,3 & 0 & 0,0 & 1 & 2,2 & 1 & 4,2 & & 3,4 & 1 & 0,6 & 3 & 1,8 \\
\hline $\begin{array}{l}\text { 2. Industriels et } \\
\text { commerçants }\end{array}$ & 1 & 2,9 & 1 & 2,6 & 0 & 0,0 & 2 & 4,3 & 5 & 11,6 & 2 & 4,6 & 7 & 16,3 & 2 & 4,8 & 2 & 8,3 & & 5,3 & 15 & 8,4 & 8 & 4,4 \\
\hline $\begin{array}{l}\text { 4. Cols blancs semi } \\
\text { et non spécialisés }\end{array}$ & 1 & 2.9 & 0 & 0,5 & 0 & 0,0 & 0 & 0,6 & 0 & 0,0 & 0 & 0,4 & 0 & 0,0 & 0 & 0,5 & 0 & 0,0 & 0 & 0,9 & 1 & 0,6 & 1 & 0,6 \\
\hline 5. Cultivateurs & 22 & 64,8 & 26 & 77,0 & 26 & 76,5 & 21 & 62,2 & 24 & 55,8 & 24 & 56,3 & 24 & 55,8 & 23 & 52,3 & 11 & 45,8 & 12 & 47,3 & 107 & 60,0 & 104 & 58,2 \\
\hline 6. Artisans & 1 & 2,9 & 0 & 1,1 & 2 & 5,9 & 1 & 1,6 & 0 & 0,0 & 1 & 1,4 & 1 & 2,3 & 1 & 1,7 & 4 & $\mid 16,7$ & 0) & 1,3 & 8 & 4,5 & 2 & 1,4 \\
\hline 7. Ouvriers spécialisés & 0 & 0,0 & 0 & 0,2 & 1 & 2,9 & 0 & 0,2 & 0 & 0,0 & 0 & 0,5 & 0 & 0,0 & 1 & 1,5 & 0 & 0,0 & 1 & 2,5 & 1 & 0,6 & 2 & 1,2 \\
\hline $\begin{array}{l}\text { 8. Gens de métier } \\
\text { (indéterminés } \\
\text { de } 6 \text { et } 7 \text { ) }\end{array}$ & 4 & 11,8 & 3 & 7,8 & 2 & 5,9 & 3 & 7,7 & 3 & 7,0 & 4 & 8,9 & 7 & 16,3 & 3 & 7,5 & 1 & 4,2 & $?$ & 8,9 & 17 & 9,5 & 15 & 8,2 \\
\hline Toutes catégories (b) & 34 & 100 & 34 & 100 & 34 & 100 & 34 & 100 & 43 & 100 & 43 & 100 & 43 & 100 & 43 & 100 & 24 & 100 & 2.4 & 100 & 178 & 100 & 178 & 100 \\
\hline
\end{tabular}

Notes: (a) Dans ce tableau, les valeurs attendues sont celles que l'on devrait obtenir si la distribution socio-professionnelle des augustines hospitalières était proportionnelle aux effectifs de chaque catégorie dans l'ensemble de la population. Ces effectifs ont été établis à partir d'un groupe-témoin formé des 19719 femmes nées et ayant contracté un premier mariage au Saguenay entre 1882 et 1947.

(b) Nombre de professions indéterminées: 8.

(SOREP) 
- Les communautés religieuses ne formaient pas un univers monolithique. De l'une à l'autre, en fonction de leurs rôles et surtout de leur implantation géographique, on observe une importante diversité dans l'orientation spatiale et sociale du recrutement. Ceci oblige le chercheur à adopter une échelle $\mathrm{d}$ 'analyse très fine.

- Les écarts relevés dans les milieux de recrutement ne paraissent pas imputables à des clivages dans la pratique religieuse et dans la dévotion. On ne voit pas ici voisiner des espaces dévots et des espaces irréligieux: l'univers de la foi était relativement uniforme.

- Jusqu'à preuve du contraire, ces écarts ne semblent pas non plus découler d'une sélection sociale délibérée, fruit d'une orientation préférentielle de la part des communautés. Même les règlements relatifs à la dot, appliqués d'une manière très flexible et sujets à toutes sortes d'accommodements, ne semblent pas avoir rempli la fonction d'exclusion qu'on est tenté de leur prêter ${ }^{13}$.

- La courbe du recrutement, qui s'incline à partir de la décennie 1932-1941, montre une cassure beaucoup plus précoce que prévu et suggère une redéfinition du calendrier des mutations qui ont affecté la vie religieuse au Saguenay — pour s'en tenir à cette région.

À la lumière de ces conclusions, notre enquête sera évidemment prolongée et ce, dans deux directions. La perspective en sera d'abord étendue, afin d'y inclure les prêtres et les religieux (pères, frères). L'analyse se portera ensuite sur les modalités familiales du recrutement, c'est-à-dire l'ensemble des paramètres qui ont le plus immédiatement déterminé les professions ecclésiastiques et religieuses (notamment: le rang de naissance, la dimension de la famille, l'alphabétisation des parents, les ruptures d'union, etc.). Pour le reste, il n'est pas possible pour l'instant de prolonger cette recherche au-delà de 1947; il faudra pour cela compléter le répertoire de Frenette, ce qui n'entre pas dans nos objectifs à court terme.

13 Cet énoncé, qui rejoint les conclusions de M. DALLAIRE (1986, notamment: chap. II-IV), s'appuie sur des données préliminaires, en cours de collecte. 


\section{Références bibliographiques}

BÉLANGER, Diane et Lucie ROZON, 1982. Les religieuses au Québec, Montréal, Éditions Libre Expression, 338 p.

BOUCHARD, Gérard et Christian POUYEZ, 1985. «Les catégories socio-professionnelles: une nouvelle grille de classement», Lahour/l.e Travail, 15 (Spring/printemps), p. 145-163.

BOUCHARD, Gérard, Raymond ROY et Bernard CASGRAIN, 1985. Reconstitution automailque des furmilies. Le système SOREP, Dossier no. 2, 2 vol., Université du Québec à Chicoutimi, septembre, $745 \mathrm{p}$.

BOUCHARD, Gérard, Raymond ROY et Bernard CASGRAIN, 1986. «De la micro à la macro-reconstitution des familles. Le système SOREP», GENUS, vol. XLII, 3-4 (décembre), p. 33-54.

BRIDE, A., 1937. «Religieux-religieuses», Dictionnaire de théologie catholique, vol. XIII-2, Paris, Letouzé et Ané, col. 2157-2182.

BRIDE, A., 1949. "Clercs», dans Catholicisme. Hier, aujourd'hui, demain, vol. II, Paris, Letouzé et Ané, col. 1209-1212.

BRIDE, A., 1967. «Laïcs», dans Catholicisme. Hier, aujourd'hui, demain, Paris, Letouzé et Ané, col. 1639-1642.

DALLAIRE, Micheline, 1986. Les dots des religieuses au Canada français, 1639-1800. Étude économique et sociale, Montréal, Éditions Hurtubise HMH, 244 p.

DANYLEWYCZ, Marta, 1987. Taking the Veil: An Alternative to Marriage, Motherhood, and Spinsterhood in Quebec, 1840-1920, Toronto, McClelland and Stewart, $195 \mathrm{p}$.

DENAULT, Bernard et Benoît LÉVESQUE, 1975. Éléments pour une sociologie des communautés religieuses au Québec, Montréal/ Sherbrooke, Les Presses de l'Université de Montréal/Université de Sherbrooke, $220 \mathrm{p}$.

DUMONT-JOHNSON, Micheline, 1978. «Les communautés religieuses et la condition féminine», Recherches sociographiques, vol. XIX, no. 1, p. 79-102.

FRENETTE, F.-X.-Eugène (chanoine), 1945. Notices biographiques et notes historiques sur le diocèse de Chicoutimi, Chicoutimi, Chancellerie de l'Évêché, 243 p.

FRENETTE, F.-X.-Eugène (chanoine), 1947. Supplément aux «Notices biographiques et notes historiques» sur le diocèse de Chicoutimi, Chicoutimi, Chancellerie de l'Évêché, 415 p. 
GUÉRIN, Michel et Gérard BOUCHARD, 1988. Statistiques de l'urbanisation au Saguenay, Université du Québec à Chicoutimi, Documents de SOREP no. II-C-143, (octobre), $10 \mathrm{p}$.

HAMELIN, Jean et Nicole GAGNON, 1984. Histoire du catholicisme québécois. Le XXe siècle. Tome 1, 1898-1940, Montréal, Les Éditions du Boréal Express, 504 p.

JEAN, Marguerite, 1977. Évolution des communautés religieuses de femmes au Canada de 1639 à nos jours, Montréal, Fides, $324 \mathrm{p}$.

JUNEAU, Danielle et Nicole LAURIN, 1986. «Les communautés religieuses de femmes au Québec. Une recherche en cours», Questions de culture, no. 9, «Identités féminines: mémoire et création» (I.Q.R.C.) p. 145-156.

LESSARD, Marc-André et Jean-Paul MONTMINY, 1967. «Les religieuses du Canada: âge, recrutement et persévérance», Recherches sociographiques, vol. VIII, 1 (janvier-avril), p. 15-47.

PELLETIER, Louis, 1986. Évolution démographique des religieux en Nouvelle-France, Mémoire présenté à la Faculté des études supérieures en vue de l'obtention de la maitrise en démographie, Université de Montréal, xii, $196 \mathrm{p}$.

POUYEZ, Christian, Yolande LAVOIE, Gérard BOUCHARD, Raymond ROY et al., 1983. Les Saguenayens. Introduction à l'histoire des populations du Saguenay, XVIe-XXe siècles, Québec, Presses de l'Université du Québec, 386 p.

SIMARD, André, 1969. Les évêques et les prêtres séculiers au diocèse de Chicoutimi 1878-1968, Chicoutimi, Chancellerie de l'Évêché, 812 p. 